\title{
Time and Cost Performance Status of Sikta Irrigation Contract
}

\author{
Anjay Kumar Mishra*, Prakash Yadav**, \& P. S. Aithal*** \\ *Post-Doctoral Research Scholar, Srinivas University, India and Associate Professor, Madan \\ Bhandari Memorial Academy Nepal, Urlabari3, Morang, Nepal \\ OrcidID: 0000-0003-2803-4918; Email: anjaymishra2000@ gmail.com \\ **Engineer, Khando River Management Project, Saptari, Nepal; E-mail: \\ prydv22@gmail.com \\ *** Professor, College of Management \& Commerce, Srinivas University, Mangalore, India \\ OrcidID: 0000-0002-4691-8736; E-mail: psaithal@gmail.com
}

Area/Section: Construction Management.

Type of the Paper: Analytical Policy Research.

Type of Review: Peer Reviewed as per $|\mathrm{C}| \mathrm{O}|\mathrm{P}| \mathrm{E} \mid$ guidance.

Indexed in: OpenAIRE.

DOI: https://doi.org/10.5281/zenodo.5081379

Google Scholar Citation: IJMTS

\section{How to Cite this Paper:}

Mishra, Anjay Kumar, Prakash Yadav \& Aithal, P. S., (2021). Time and Cost Performance Status of Sikta Irrigation Contract. International Journal of Management, Technology, and Social Sciences (IJMTS), 6(1), 286-305. DOI: https://doi.org/10.5281/zenodo.5081379.

International Journal of Management, Technology, and Social Sciences (IJMTS)

A Refereed International Journal of Srinivas University, India.

CrossRef DOI: https://doi.org/10.47992/IJMTS.2581.6012.0145

(C) With Author.

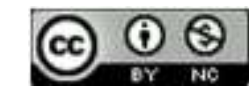

This work is licensed under a Creative Commons Attribution-Non-Commercial 4.0 International License subject to proper citation to the publication source of the work.

Disclaimer: The scholarly papers as reviewed and published by the Srinivas Publications (S.P.), India are the views and opinions of their respective authors and are not the views or opinions of the SP. The SP disclaims of any harm or loss caused due to the published content to any party. 


\title{
Time and Cost Performance Status of Sikta Irrigation Contract
}

\author{
Anjay Kumar Mishra*, Prakash Yadav**, \& P. S. Aithal*** \\ *Post-Doctoral Research Scholar, Srinivas University, India and Associate Professor, Madan \\ Bhandari Memorial Academy Nepal, Urlabari3, Morang, Nepal \\ OrcidID: 0000-0003-2803-4918; Email: anjaymishra2000@ gmail.com \\ **Engineer, Khando River Management Project, Saptari, Nepal; E-mail: \\ prydv22@gmail.com \\ *** Professor, College of Management \& Commerce, Srinivas University, Mangalore, India \\ OrcidID: 0000-0002-4691-8736; E-mail: psaithal@gmail.com
}

\begin{abstract}
Purpose: The government of Nepal (GoN) is implementing many small, medium, and large types of Irrigation Projects. Sikta Irrigation Project (SIP) is the National Pride Project implemented by the GoN. The command area of the project has 42766.00 ha and beneficiaries of the project have 46715 households consisting of 449588 population of Banke district. The paper aims to assess the contract performance in terms of the Time and Cost of the Sikta Irrigation Project, Banke, Nepal.

Design/Methodology/Approach: Using secondary data of contract, content Analysis of Project documents review and Key Informant Interviews were used for assessing the contract performance.

Findings/Result: Sikta Irrigation Project has a total of 52 contracts out of which consider for study based on documentation, only 18 contracts completed based on schedule time whereas 16 contracts behind schedule. However, 16 contracts are still on going far behind the schedule and one contract terminated. The actual expenditure of the project has 16.26 billion $(64.90 \%$ of estimated cost) and the allocated budget was 18.26 billion (72.99\% of estimated cost) whereas the estimated cost has 25.032 billion. It indicates that the progress of the project was $64.90 \%$ and fails to complete the scheduled time 2076/077. The client should prepare the project well before implementation with proper planning, designing, and detailed study from the beginning.
\end{abstract}

Originality/Value: It is empirical research to assure the project performance based on lessons from Sikta Irrigation Project.

Paper Type: Analytical Policy Research

Keywords: Irrigation, contract, expenditure, Contract Duration, Performance

\section{INTRODUCTION :}

Nepal has sufficient water resources. That is why the Government of Nepal has a high priority in the irrigation sector. In Nepal, the National pride projects in the irrigation sector are Sikta Irrigation Project, Babai Irrigation Project, Rani-Jamar-Kularia Irrigation Project, Bheri-Babai Diversion Multipurpose Project, and Sunkoshi Merin Multipurpose Project.

According to Mishra et al, (2021) [1], Sikta Irrigation Project was selected for the study purpose. It is located in the Banke district. The water source for this project is West Rapti River, which originates from the mid-mountains in the Mid-Western Region of the country. There are two system of canal i.e., Eastern canal command area 9000ha and western canal command area 33766 ha. When project have been completed then beneficiary of the project has 46715 household and 449588 population of Banke district. Neediness, lack of education and so forth are seen in Banke locale, where Sikta Irrigation Project is under development. The general objective of the Sikta water system project is to add to the National Development destinations of the Government. The arrangement of water system offices along with the improvement of horticultural, support administrations which would work on the useful limit of ranchers so agrarian creation will be expanded and expectation for everyday comforts of the ranchers 
in Banke area will be upgraded. A significant test of the task is the dispersive soil structure in the trench. The disappointment of trench at the hour of trail testing is an illustration of specialized issue. The dispersive soil is seen where the trench and different designs were built. As per the master, cutting of existing soil and filling by sweet soil is essential before the development of the trench and other design according to the necessity of the site state of the task. Huge volume of soil substitution is seen which will diminish the issue. The project performance is measured base on timely completion, within the budget, required quality standards and customers satisfaction (Mishra and Bhandari, 2018) [2]. As a consequence of poor performance, the projects mainly face the problem of time overrun and cost overrun. It is always discussed to complete project on time within budget using Value Management and even crashing could be done if cost allows but in practice time extension is norms not an exception in Nepal [3].

Sikta Irrigation project of command area 42766 ha and beneficiary of the project have 46715 house hold and 449588 population of Banke district [1]. The delay of project might affect agriculture productivity. A country like Nepal where food security and food severance are big issue need to focus on its agricultural development, on agricultural infrastructure. In this circumstance this project itself is unique one to increase the yield of agriculture product in the area to overcome starvation significantly in the respected area that's why the project should have been completed on time with accepted quality within the budget without any safety or ethically issues so the researcher has attempt to conduct the study and contractual performance assessment of Sikta Irrigation Project.

Sikta Irrigation Project was started 2004/2005 (B.S.2061/062) [4] with an objective to complete by 2019/2020 (B.S.2076/077) however the project has not completed yet so its performance needs to be study and issue related performance should be accessed so the researcher as made an attempt to continue to conduct an area.

\section{OBJECTIVES :}

The objective of the study is to assess the status of Contact in terms of time and cost of Sikta Irrigation Project.

\section{SILENT FEATURE OF SIKTA IRRIGATION PROJECT :}

The concept for the SIP was first formulated in prefeasibility studies conducted from 1975 to 1976 and contained in the report on the proposed Western Rapti Multipurpose Development, which comprised the construction of a high dam at Bhalubang in the West Rapti Basin and ad The German company Lahmeyer International GmbH completed a feasibility study for SIP in August 1980 based on a run-of -the-river diversion gravity irrigation scheme. A further study for a run-of-the-river scheme was carried out by the department of Irrigation, Hydrology and Metrology and this was completed in June 1983. In 2004, Feasibility report was assessed.

The study showed that the project is technically, economically and socially viable in the present context as well. Based on the Feasibility Study Report 2004, the Government of Nepal decided to implement the Project in three phases, which are as follows. The total cost of the Project as per the Study is NRS 7.45 billion (revised to NRs 12.80 billion in Fiscal Year 2007/08). Later second revision and add scope of work the approved total financial cost of whole Project was NRS. 25.02 billion in fiscal year 2071/72 B.S. However, the cost of the Project has been increased to NRs 39.98 billion due to rectification of right main canal, some additional command area development and command area protection works, increased cost of Left main canal as per contract agreement, increased cost of Dunduwa irrigation system as per DPR, social cost to address social requirements of project affected area, increased cost of land acquisition, and inflation (SIP Master Plan, 2019) [5].

Table 1 : Silent Feature of SIP

\begin{tabular}{|l|l|l|}
\hline 1 & Name of Project & Sikta Irrigation Project \\
\hline 2 & Location & Provenance no- 5, Banke, Nepal \\
\hline 4 & Culturable Command Area & 33766 Ha. (Western), 9000 Ha. (Eastern) \\
\hline
\end{tabular}




\begin{tabular}{|c|c|c|}
\hline 5 & Name of Source River & West Rapti \\
\hline 6 & Type of Source & Prennial \\
\hline 7 & Catchment Area & $5450 \mathrm{~km}^{2}$ \\
\hline 8 & Minimum Monthly Flow Rate & 12.5 cumecs ( $80 \%$ of Reliable flow) \\
\hline 9 & High Flood Design Discharge & 8659 Cumecs (100years return period) \\
\hline 10 & Diversion Structure & Barrage, $317 \mathrm{~m}$ (Dam length) \\
\hline \multirow{2}{*}{11} & \multirow{2}{*}{ Span No and Length } & Barrage portion-13 no@17m span gates \\
\hline & & $\begin{array}{l}\text { Under sluice portion - Right Side: } 3 \text { no @ 10m span gates, Left } \\
\text { Side: 2no @ 10m span gates }\end{array}$ \\
\hline 12 & Desalting Basin Length & Right Side -640m, Left Side- \\
\hline 13 & Western Canal & Total Length: $45.25 \mathrm{~km}$, Ideal Length:30km, Branch-7 no \\
\hline 14 & Eastern Canal & Total Length: $53 \mathrm{~km}$, Ideal Length: $1.45 \mathrm{~km}$, Branch-2 no \\
\hline 15 & Discharge of Canal & Western :50 cumecs, Eastern: 12.50 cumecs \\
\hline 16 & Beneficiary Population & 449588 \\
\hline 17 & Beneficiary Household & 46715 \\
\hline 18 & Present Cropping Intensity & $168 \%$ \\
\hline 19 & Future Cropping Intensity & $242 \%$ \\
\hline 20 & Project Start & $2061 / 062$ \\
\hline 21 & Project complete & 2076/077 (Based on Revised Master Plan 2014) \\
\hline 22 & Total Project Cost & 25.02 billion \\
\hline
\end{tabular}

(Source: SIP Revised Master Plan, 2014) [5].

\section{LITERATURE REVIEW :}

\section{Time and Cost performance of Construction Project}

Time overrun is norms not an exception in Nepalese case for construction projects. Time overrun resulted into cost overrun. All the projects under Second Small-Town Water Supply and Sanitation Projects selected for study were time overrun (Mishra et al, 2020) [6]. According to Mishra et.al. [7], the project time and cost of the Public Health Buildings projects in Nepal was highly correlated ( $\mathrm{p}=$ 0.599). An equation, Time $=487.5 \times(\mathrm{C} / 79.96) 0.293$ (time is expressed in day and cost in million NRs.) was developed for the prediction of time and cost components of similar projects. The venture complete time-cost relationship can be dictated by including the immediate expense and circuitous expense esteems together. The ideal undertaking term can be resolved as the task span that outcomes at all venture all out cost. As indicated by Menon et al., [8] appropriate arranging of work, submitted authority and the board, and powerful correspondence framework can be exceptionally useful in further developing time execution. To limit the defers Suwal et al., [9] suggested following that (I) there ought to be arrangements in PPA and PPR to control uncommon low bid by expanding execution security according to level of bid underneath assessed sum, (ii) Appropriate workers for hire ought to be chosen dependent on experience, specialized, monetary limit alongside thought of work close by. The 
arrangement of negative stamping according to work close by ought to be done in Public Procurement Act (PPA) and Public Procurement Rules (PPR), with the goal that propensity of possessing works will be diminished, (iii) Pre-execution readiness, (for example, land obtaining, utility migration, EIA and IEE) and arranging of undertaking assignments, asset need and suitable agreement methodology to keep away from low bid ought to be done, (iv) Strict arrangements or limit on project period varieties ought to be done in PPA and PPR, (v) Proper component ought to be developed for steady and fixed residency of Project supervisor in the venture, (vi) Efficient agreement the executives by manager (fast choice and endorsement on schedule).

The project delay is still happening and will continue to happen in the construction for various known and unknown reasons such as unexpected factors, bankruptcy from client, and change in design during the construction, political issue, sudden price fluctuation, with projects manager in result of forcing $\mathrm{him} /$ her to leave before completing the assigned job. However, time and cost overrun may not be prevented entering but the evolving new technology like BIM, new methods and past experience could be used to reduce the impact from recognized risk factors, particularly in the undeveloped and developing countries (Yadav and Mishra) [10]. Time Overrun is one of the most significant issues being faced by the construction industry today also.

\section{RESEARCH METHODOLOGY :}

\subsection{Selection of Study Area:}

Sikta Irrigation Project is the National Pride Project, it is located at the Banke District. The water source for this project is West Rapti River, which originates from the mid-mountains in the Mid-Western Region of the country. The source of this irrigation system is prennial. There are two system of canal i.e., Eastern canal and Western canal. Eastern canal passes through Rapti Sonari Gaupalika and Narainapur Gaupalika its length $53.50 \mathrm{~km}$ and command area 9000 ha. There are 11 no's of secondary canal. Among them Rajkulo contains S1, S2, S3, S4 and Phattepur contains S5, S6, S7, S8, S9, S10, S11. The length of secondary and tertiary canal is $145 \mathrm{~km}$. The discharge of Eastern canal 14 cumec. Western canal passes through Rapti Sonari Gaupalika, Dudwa Gaupalika, Kohalpur Nagarpalika and Baijnath Gaupalika. Its length is $45.25 \mathrm{~km}$ with trapezoidal canal section having the tail escape at the end and command area $33766 \mathrm{~km}$. The discharge of western canal 50 cumec. There are 7 no's of secondary canal. S1 (Sidhaniya branch), Dunduwa, S2 (Gohawa), S3 (Akalgherwa), S4 (Persenpur), S5 (Pidari), S6 (GaruwaGaun) are the secondary canal. The length of secondary and tertiary canal $233 \mathrm{~km}$. The ideal length of canal is $30 \mathrm{~km}$. The bed slope of canal is $1 / 7000$. when project have been completed then beneficiary of the project has 46715 house hold and 449588 population of Banke district.

Sikta, which started construction 14 years ago in magh 2063. It was supposed to be built at an average cost of Rs 12 billion. Five years after scheduled time, the project has started partially providing water to the farmers. The estimated cost of the project in the initial year 2061/062 was 12.80 billion. Later second revision and add scope of work the approved total financial cost of whole Project was NRS. 25.02 billion in fiscal year 2071/72. However, the cost of the Project has been increased to NRs 39.98 billion due to rectification of right main canal, some additional command area development and command area protection works, increased cost of Left main canal as per contract agreement, increased cost of Dunduwa irrigation system as per DPR, social cost to address social requirements of project affected area, increased cost of land acquisition, and inflation. (Source: SIP DPR, 2014) [11].

\subsection{Design and Data Collection:}

The research is ex-post facto research-based field performance as documentary study. The study depends on secondary data of contract documents and project documents. Content Analysis is the main data analysis technique as narrative from Key Informant Interview using snowball technique.

\subsection{Data Analysis:}

Content Analysis is the methods adopted. The research is a documentation of performance in terms of time and cost using narrative technique from key informant.

\subsection{Validity and Reliability:}


This is documentation based on authorized project documents after further clarification during KII. Triangulation among respondents and logical comparison of documents verification gives validity of research and comparison with literature provides reliability.

\section{RESULTS AND DISCUSSION :}

\subsection{Status of the Contract under the Project:}

The project was started 2063/064 and has a 9-year complication time i.e., 2072/073. The project got affected by several factors resulting in increased time and cost.

\subsection{Fluctuation of cost based on Approved Master plan 2007 and 2014:}

The total cost of the Project as per the study is NRS 7.45 billion (revised to NRs 12.80 billion in Fiscal Years 2007/08). Later second revision and add a scope of work the approved total financial cost of the whole Project was NRs. 25.02 billion in the fiscal year 2071/72. However, the cost of the project has been increased to NRs 39.98 billion due to rectification of the right main canal, some additional command area development and command area protection works, increased cost of Left main canal as per contract agreement, increased cost of Dunduwa irrigation system as per DPR, social cost to address social requirements of project affected area, increased cost of land acquisition, and inflation [5]. The total expenditure at end of F.Y. 2076/077 has Rs16261946890.00.

From Table 2 it is seen that the master plan 2007 total project cost was NRs.12801739074.00. After revised in 2014 the total project cost was increased to NRs 25023180198.00 due to increased scope of work and price escalation. In the 2014 revision of the master plan various scope of work was added such as Eastern Main canal, Eastern branches, detailed breakdown of Right Main branch canals, Command Development works and head work construction. In this comparison, it was seen that in master plan 2007 was not proper detailed planning due to this reason price can be increased and the scope of work was changed.

Table: 2 Comparison of Approved Master plan 2007 and 2014 [5]

\begin{tabular}{|c|c|c|c|c|}
\hline S.N. & $\begin{array}{l}\text { Description of } \\
\text { Works }\end{array}$ & $\begin{array}{l}\text { Estimated Cost as } \\
\text { per Approved } \\
\text { Master plan } \\
\text { 2007(NRs.) } \\
\end{array}$ & $\begin{array}{c}\text { Updated Cost } \\
\text { in NRs. as per } \\
\text { Approved } \\
\text { Master plan } \\
2014 \\
\end{array}$ & Analysis \\
\hline $\mathbf{A}$ & \multicolumn{4}{|l|}{ Civil Work Cost : } \\
\hline 1 & \begin{tabular}{|l|} 
Head work \\
construction
\end{tabular} & $2,591,638,724.00$ & $\begin{array}{c}2,873,398,400.0 \\
8 \\
\end{array}$ & $\begin{array}{l}\text { cost is increased due to price } \\
\text { escalation }(10.87 \%)\end{array}$ \\
\hline 2 & $\begin{array}{l}\text { Main Canal } \\
\text { construction } \\
\text { (RMC) }\end{array}$ & $4,247,722,000.00$ & $\begin{array}{c}5,679,667,364.3 \\
4\end{array}$ & $\begin{array}{l}\text { cost is increased due to price } \\
\text { escalation and increased } \\
\text { scope of work like length of } \\
\text { canal lining, local drainage } \\
\text { structures }(33.7 \%)\end{array}$ \\
\hline \multirow[t]{4}{*}{3} & Branch Canals & $1,090,664,145.00$ & & Break down under 7 canals \\
\hline & Sidhaniya B C & - & $320,208,997.16$ & $\begin{array}{l}\text { Detailed cost breakdown of } \\
\text { branch canals was not } \\
\text { included in previously } \\
\text { approved Master Plan }\end{array}$ \\
\hline & Dunduwa B C & & $\begin{array}{c}1,529,078,523.6 \\
6\end{array}$ & - \\
\hline & \begin{tabular}{|l|} 
Other Branch \\
Canal of RMC (5 \\
Nos) \\
\end{tabular} & - & $\begin{array}{c}1,110,638,419.3 \\
3\end{array}$ & $171 \%$ increased \\
\hline 5 & Left Main Canal & - & $1,604,664,821$ & $\begin{array}{l}\text { Scope of work has been } \\
\text { changed to included left side } \\
\text { command area in } 9 \text { VDC's } \\
(100 \%)\end{array}$ \\
\hline
\end{tabular}




\begin{tabular}{|c|c|c|c|c|}
\hline 6 & $\begin{array}{l}\text { Branch Canal of } \\
\text { LMC }\end{array}$ & - & $39,443,184$ & $\begin{array}{l}\text { Scope of work has been } \\
\text { changed to included left side } \\
\text { command area in 9 VDC's } \\
(100 \%)\end{array}$ \\
\hline 7 & CAD Works & - & $2,169,289,855$ & $\begin{array}{l}\text { The cost of CAD work was } \\
\text { not included in previously } \\
\text { approved Master Plan }\end{array}$ \\
\hline 8 & $\begin{array}{l}\text { Command Area } \\
\text { Protection Works }\end{array}$ & $112,853,251$ & $540,637,390$ & $\begin{array}{l}\text { Cost is increased due to } \\
\text { price escalation and } \\
\text { increased scope of works }\end{array}$ \\
\hline & Sub-total (A) & $8,042,878,120$ & $15,867,026,955$ & (97.28\% increased) \\
\hline B & \multicolumn{4}{|l|}{ Program cost : } \\
\hline 9 & $\begin{array}{l}\text { Consultancy } \\
\text { services }\end{array}$ & $207,146,000$ & $271,849,275$ & $\begin{array}{l}\text { cost is increased as scope of } \\
\text { work has been changed to } \\
\text { include left side command } \\
\text { area in } 9 \text { VDC and due to } \\
\text { price escalation. }(31.23 \%)\end{array}$ \\
\hline 10 & $\begin{array}{l}\text { Agricultural } \\
\text { stretching support } \\
\text { program }\end{array}$ & $113,910,000$ & $117,525,362$ & $\begin{array}{l}\text { cost is increased as scope of } \\
\text { work has been changed to } \\
\text { include left side command } \\
\text { area in } 9 \text { VDC and due to } \\
\text { price escalation }(10.88 \%)\end{array}$ \\
\hline 11 & \begin{tabular}{|l} 
Institutional \\
Development/ \\
Training \\
\end{tabular} & $39,000,000$ & $13,043,478$ & - \\
\hline 12 & \begin{tabular}{|l|} 
Environmental \\
impact mitigation \\
\end{tabular} & $41,000,000$ & $42,301,449$ & $\begin{array}{l}\text { Cost is increased due to price } \\
\text { escalation. }(3.17 \%)\end{array}$ \\
\hline & Sub-total $(B)$ & $401,056,000$ & $444,719,565$ & (10.88\% increased) \\
\hline $\mathbf{C}$ & \multicolumn{4}{|l|}{ Other costs: } \\
\hline 13 & Land acquisition & $411,800,000$ & $1,601,449,275$ & $\begin{array}{l}\text { cost is increased as scope of } \\
\text { work has been changed to } \\
\text { include left side command } \\
\text { area in } 9 \text { VDC and due to } \\
\text { price escalation }(288.89 \%)\end{array}$ \\
\hline 14 & \begin{tabular}{|l|}
$\begin{array}{l}\text { Vehicle and } \\
\text { equipment cost }\end{array}$ \\
\end{tabular} & $28,200,000$ & $44,147,101$ & \begin{tabular}{|l} 
Cost is increased due to price \\
escalation $(56.55 \%)$
\end{tabular} \\
\hline \multirow[t]{6}{*}{15} & Establishment & & & \\
\hline & Furniture & $29,000,000$ & 765,942 & Cost is decreased by $-2.64 \%$ \\
\hline & Machinery goods & & $5,217,391$ & $\begin{array}{l}\text { Cost is increased due to price } \\
\text { escalation }(100 \%)\end{array}$ \\
\hline & $\begin{array}{l}\text { Building } \\
\text { construction/ } \\
\text { maintenance } \\
\end{array}$ & & $29,920,290$ & $\begin{array}{l}\text { Cost is increased due to price } \\
\text { escalation }(100 \%)\end{array}$ \\
\hline & Sub-total (C) & $469,000,000$ & $1,681,500,000$ & Cost increased by $258.52 \%$ \\
\hline & Total Base Cost & $8,912,934,120$ & $17,993,246,521$ & Cost increased by $101.87 \%$ \\
\hline $\mathbf{D}$ & \multicolumn{4}{|c|}{ Contingencies \& VAT: } \\
\hline 16 & $\begin{array}{l}\text { D1. Work Charge } \\
\text { Staff Cost and } \\
\text { Other Minor } \\
\text { Expenses } \\
\end{array}$ & 449562406 & $899,662,326$ & $\begin{array}{l}5 \% \text { of Base course }(100.12 \% \\
\text { increased) }\end{array}$ \\
\hline 17 & \begin{tabular}{|l|} 
D2. Physical \\
Contingencies \\
\end{tabular} & $944,081,053$ & $1,799,324,652$ & $\begin{array}{l}10 \% \text { of Base Course } \\
(90.59 \% \text { increased })\end{array}$ \\
\hline
\end{tabular}




\begin{tabular}{|l|l|c|c|l|}
18 & $\begin{array}{l}\text { D3. Price } \\
\text { escalation local } \\
\text { cost }\end{array}$ & $1,472,766,442$ & $1,799,324,652$ & $\begin{array}{l}10 \% \text { of Base course }(22.17 \% \\
\text { increased })\end{array}$ \\
\hline 19 & VAT & $944,081,053$ & $\begin{array}{c}2,339,122,047.6 \\
9\end{array}$ & $\begin{array}{l}13 \% \text { of Base course } \\
(147.76 \% \text { increased })\end{array}$ \\
\hline & sub-total(D) & $3,810,490,954$ & $\begin{array}{c}6,837,433,677.8 \\
6\end{array}$ & Cost increased by $79.44 \%$ \\
\hline E & $\begin{array}{l}\text { Administrative } \\
\text { expenditure }\end{array}$ & $78,314,000$ & $192,500,000.00$ & Cost increased by $145.80 \%$ \\
\hline \multicolumn{2}{|l}{ Total Project Cost } & $\mathbf{1 2 , 8 0 1 , 7 3 9 , 0 7 4}$ & $\begin{array}{c}\mathbf{2 5 , 0 2 3 , 1 8 0 , 1 9 8} . \\
\mathbf{5 4}\end{array}$ & Cost increased by $95.47 \%$ \\
\hline
\end{tabular}

From table 2 it is seen that comparison of Master plan 2007 and Master plan 2014, civil work cost of project has increased by $97.28 \%$, program cost increased by $10.88 \%$, other cost increase by $258.52 \%$, contingencies \& Vat $79.44 \%$ and administrative expenditure increased by $145.80 \%$ because of increase in the scope of work in this item and increase in price index. The price index of the Rastriya Bank in 2007 was 74.9 and 2014 was 141.3 i.e., increased by $88.65 \%$. The total cost of the Project has increased by $95.47 \%$. It was clearly saying that due to improper planning of project cause to increase in total Project cost.

\subsection{Assessment of Schedule Contract:}

Out of a total of 52 contracts, only 18 contracts were completed based on schedule time whereas 16 contracts behind the schedule. However, 16 contracts are still on going far behind the schedule. The status of timely completed 18 contract's which estimated cost, agreement amount and expenditure are shown in table 3.0

Table 3: Contracts completed based on Schedule

\begin{tabular}{|c|c|c|c|c|c|c|c|c|c|}
\hline $\begin{array}{l}\text { S. } \\
\text { N. }\end{array}$ & $\begin{array}{l}\text { Stretch of } \\
\text { Canal }\end{array}$ & $\begin{array}{l}\text { Contract } \\
\text { No }\end{array}$ & $\begin{array}{l}\text { Name of the } \\
\text { contractor }\end{array}$ & $\begin{array}{l}\text { Estima } \\
\text { ted } \\
\text { Amou } \\
\text { nt }\end{array}$ & $\begin{array}{l}\text { Agree } \\
\text { ment } \\
\text { Amoun } \\
\text { t }\end{array}$ & $\begin{array}{l}\text { Total } \\
\text { Expen } \\
\text { diture }\end{array}$ & $\begin{array}{l}\text { Date of } \\
\text { signing } \\
\text { of } \\
\text { contra } \\
\text { ct }\end{array}$ & $\begin{array}{l}\text { Sched } \\
\text { ule } \\
\text { Date } \\
\text { of } \\
\text { compl } \\
\text { etion }\end{array}$ & $\begin{array}{l}\text { Comp } \\
\text { leted } \\
\text { date }\end{array}$ \\
\hline 1 & $\begin{array}{l}\text { 1400m } \\
\text { length } \\
\text { Desilting } \\
\text { Basin, } \\
317 \mathrm{~m} \\
\text { Weir cum } \\
\text { Bridge, } \\
\text { Flushing } \\
\text { Channel } \\
\& \\
\text { Protection } \\
\text { work }\end{array}$ & $\begin{array}{l}\mathrm{SIP} / \mathrm{HW} / \mathrm{IC} \\
\mathrm{B}-01\end{array}$ & $\begin{array}{l}\text { SINOHYDRO } \\
\text { Corporation } \\
\text { Chania and } \\
\text { Lumbini } \\
\text { Builders JV. }\end{array}$ & $\begin{array}{l}233649 \\
9000\end{array}$ & $\begin{array}{l}185505 \\
1000\end{array}$ & $\begin{array}{l}185420 \\
0000\end{array}$ & $\begin{array}{l}2006- \\
06-05\end{array}$ & $\begin{array}{l}2010- \\
05-11\end{array}$ & $\begin{array}{l}2010- \\
05-10\end{array}$ \\
\hline 2 & $\begin{array}{l}\text { ch } 15+\mathrm{km} \\
\text { to ch } \\
16+000 \\
\mathrm{~km}\end{array}$ & $\begin{array}{l}\text { SIP/MC/N } \\
\mathrm{CB} / 04\end{array}$ & $\begin{array}{l}\text { P.S/Golden/Bha } \\
\text { irav } \\
\text { Construction (p) } \\
\text { Ltd. }\end{array}$ & $\begin{array}{l}109856 \\
000\end{array}$ & $\begin{array}{l}891252 \\
60.21\end{array}$ & $\begin{array}{l}890000 \\
00\end{array}$ & $\begin{array}{l}2011- \\
11-06\end{array}$ & $\begin{array}{l}2013- \\
11-05\end{array}$ & $\begin{array}{l}2013- \\
11-05\end{array}$ \\
\hline 3 & $\begin{array}{l}\text { Ch. } 16+00 \\
\mathrm{~km} \text { to ch } \\
17+000 \\
\mathrm{~km}\end{array}$ & $\begin{array}{l}\mathrm{SIP} / \mathrm{MC} / \mathrm{N} \\
\mathrm{CB} / 05\end{array}$ & $\begin{array}{l}\text { Siddhi sai } \\
\text { Prakash (p) } \\
\text { Ltd., Mahalaxmi } \\
\text { construction (p) } \\
\text { Ltd. \& Mahadev } \\
\text { Khimti Nirman } \\
\text { Sewa (P)Ltd. }\end{array}$ & $\begin{array}{l}119894 \\
000\end{array}$ & $\begin{array}{l}969014 \\
49.8\end{array}$ & $\begin{array}{l}960000 \\
00\end{array}$ & $\begin{array}{l}2011- \\
11-06\end{array}$ & $\begin{array}{l}2013- \\
11-05\end{array}$ & $\begin{array}{l}2013- \\
11-05\end{array}$ \\
\hline
\end{tabular}




\begin{tabular}{|c|c|c|c|c|c|c|c|c|c|}
\hline 4 & $\begin{array}{l}\text { Ch. } 17+00 \\
0 \mathrm{~km} \text { to } \\
\text { ch } \\
17+700 \\
\mathrm{~km}\end{array}$ & $\begin{array}{l}\text { SIP/MC/N } \\
\mathrm{CB} / 06\end{array}$ & $\begin{array}{l}\text { Hirachan } \\
\text { construction (p) } \\
\text { Ltd. \& Mahadev } \\
\text { khimti Nirman } \\
\text { Sewa (p) Ltd. \& } \\
\text { anjan Nirman } \\
\text { Sewa (p)Ltd. }\end{array}$ & $\begin{array}{l}861937 \\
50.8\end{array}$ & $\begin{array}{l}861937 \\
50.81\end{array}$ & $\begin{array}{l}860000 \\
00\end{array}$ & $\begin{array}{l}2011- \\
11-06\end{array}$ & $\begin{array}{l}2013- \\
11-05\end{array}$ & $\begin{array}{l}2013- \\
11-05\end{array}$ \\
\hline 5 & $\begin{array}{l}\text { Constructi } \\
\text { on of } \\
\text { Canal } \\
\text { Lining, } \\
\text { Drain \& } \\
\text { Protection } \\
\text { Work }\end{array}$ & $\begin{array}{l}\text { SIP/MC/N } \\
\text { CB-07 }\end{array}$ & $\begin{array}{l}\text { Kalika } \\
\text { Construction P } \\
\text { Ltd }\end{array}$ & $\begin{array}{l}390106 \\
81.9\end{array}$ & $\begin{array}{l}354321 \\
48.69\end{array}$ & $\begin{array}{l}319572 \\
44\end{array}$ & $\begin{array}{l}2015- \\
03-15\end{array}$ & $\begin{array}{l}2017- \\
02-11\end{array}$ & $\begin{array}{l}2017- \\
02-11\end{array}$ \\
\hline 6 & $\begin{array}{l}\text { Aagiya } \\
\text { Camp } \\
\text { compound } \\
\text { wall and } \\
\text { main } \\
\text { canal } \\
\text { lining } \\
\text { works }\end{array}$ & $\begin{array}{l}\text { SIP/MC/H } \\
\text { W/NCB-01 }\end{array}$ & $\begin{array}{l}\text { Swachchhanda, } \\
\text { Gongabu, } \\
\text { Kathmandu }\end{array}$ & $\begin{array}{l}124265 \\
42.6\end{array}$ & $\begin{array}{l}124265 \\
42.62\end{array}$ & $\begin{array}{l}121926 \\
30.00\end{array}$ & $\begin{array}{l}2016- \\
03-06\end{array}$ & $\begin{array}{l}2018- \\
03-14\end{array}$ & $\begin{array}{l}2018- \\
03-14\end{array}$ \\
\hline 7 & $\begin{array}{l}\text { Mul badh } \\
\text { tahta } \\
\text { baijapur } \\
\text { command } \\
\text { area } \\
\text { protection } \\
\text { works }\end{array}$ & $\begin{array}{l}\text { SIP/CAP/N } \\
\text { CB-01 }\end{array}$ & $\begin{array}{l}\text { Swachchhanda/ } \\
\text { Gajurmukhi JV, } \\
\text { Gongabu, } \\
\text { Kathmandu }\end{array}$ & & $\begin{array}{l}610469 \\
21.21\end{array}$ & $\begin{array}{l}599437 \\
06.00\end{array}$ & $\begin{array}{l}2015- \\
02-16\end{array}$ & $\begin{array}{l}2016- \\
07-13\end{array}$ & $\begin{array}{l}2016- \\
07-13\end{array}$ \\
\hline 8 & $\begin{array}{l}\text { Jhijra } \\
\text { Command } \\
\text { area } \\
\text { protection } \\
\text { works }\end{array}$ & $\begin{array}{l}\text { SIP/CAP/N } \\
\text { CB-04 }\end{array}$ & $\begin{array}{l}\text { DEV \& } \\
\text { SAYAR } \\
\text { MALLA JV }\end{array}$ & $\begin{array}{l}287360 \\
43.3\end{array}$ & $\begin{array}{l}249089 \\
41.43\end{array}$ & $\begin{array}{l}193464 \\
35.00\end{array}$ & $\begin{array}{l}2017- \\
06-17\end{array}$ & $\begin{array}{l}2018- \\
03-12\end{array}$ & $\begin{array}{l}2018- \\
03-17\end{array}$ \\
\hline 9 & $\begin{array}{l}\text { Paruwa } \\
\text { Command } \\
\text { area } \\
\text { protection } \\
\text { works }\end{array}$ & $\begin{array}{l}\text { SIP/CAP/N } \\
\text { CB-05 }\end{array}$ & $\begin{array}{l}\text { AMAR } \\
\text { CONST. PVT } \\
\text { LTD }\end{array}$ & $\begin{array}{l}287312 \\
64.5\end{array}$ & $\begin{array}{l}281341 \\
75.00\end{array}$ & $\begin{array}{l}266793 \\
32.00\end{array}$ & $\begin{array}{l}2017- \\
06-17\end{array}$ & $\begin{array}{l}2018- \\
03-18\end{array}$ & $\begin{array}{l}2018- \\
03-18\end{array}$ \\
\hline 10 & $\begin{array}{l}\text { Constructi } \\
\text { on of } \\
\text { Revetmen } \\
t \text { and Spur } \\
\text { at } \\
\text { Muguwa } \\
\text { Khola in } \\
\text { Hariharpu } \\
\text { r }\end{array}$ & $\begin{array}{l}\text { SIP/CAP/N } \\
\text { CB-09(Re) }\end{array}$ & $\begin{array}{l}\text { M/S AMAR / } \\
\text { MAJDOOR JV }\end{array}$ & $\begin{array}{l}214430 \\
69\end{array}$ & $\begin{array}{l}164320 \\
19.75\end{array}$ & $\begin{array}{l}150571 \\
37.00\end{array}$ & $\begin{array}{l}2018- \\
07-06\end{array}$ & $\begin{array}{l}2019- \\
07-05\end{array}$ & $\begin{array}{l}2019- \\
07-05\end{array}$ \\
\hline 11 & $\begin{array}{l}\text { River } \\
\text { Training } \\
\text { Works at } \\
\text { Harkundi } \\
\text { Khola at } \\
\text { Chainage } \\
8+400 \text { of } \\
\text { Western } \\
\text { Main } \\
\text { Canal }\end{array}$ & $\begin{array}{l}\text { SIP/CAP/N } \\
\text { CB-10(Re) }\end{array}$ & $\begin{array}{l}\text { M/S ROYAL } \\
\text { BUILDERS } \\
\text { PVT. LTD. }\end{array}$ & $\begin{array}{l}282271 \\
87\end{array}$ & $\begin{array}{l}240097 \\
62.17\end{array}$ & $\begin{array}{l}243466 \\
52.00\end{array}$ & $\begin{array}{l}2018- \\
07-05\end{array}$ & $\begin{array}{l}2019- \\
07-05\end{array}$ & $\begin{array}{l}2019- \\
07-05\end{array}$ \\
\hline
\end{tabular}




\begin{tabular}{|c|c|c|c|c|c|c|c|c|c|}
\hline 12 & $\begin{array}{l}\text { Constructi } \\
\text { on of } \\
\text { Embankm } \\
\text { ent \& } \\
\text { Protection } \\
\text { works at } \\
\text { Jharna } \\
\text { khola in } \\
\text { Tikulipur }\end{array}$ & $\begin{array}{l}\text { SIP/CAP/N } \\
\text { CB-11 }\end{array}$ & $\begin{array}{l}\text { M/S KHADKA } \\
\text { NIRMAN } \\
\text { SEWA }\end{array}$ & $\begin{array}{l}154460 \\
00\end{array}$ & $\begin{array}{l}101450 \\
00.00\end{array}$ & & $\begin{array}{l}8,378,0 \\
63.00\end{array}$ & $\begin{array}{l}11- \\
\text { Jan-18 }\end{array}$ & $\begin{array}{l}2018- \\
01-11\end{array}$ \\
\hline 13 & $\begin{array}{l}\text { Command } \\
\text { area } \\
\text { protection } \\
\text { work at } \\
\text { Paruwa } \\
\text { khola }\end{array}$ & $\begin{array}{l}\text { SIP/CAP/N } \\
\text { CB-14 }\end{array}$ & $\begin{array}{l}\text { M/S P.S. } \\
\text { NIRMAN } \\
\text { SEWA }\end{array}$ & $\begin{array}{l}118200 \\
00\end{array}$ & $\begin{array}{l}818100 \\
0.00\end{array}$ & $\begin{array}{l}7,437,1 \\
15.00\end{array}$ & $\begin{array}{l}2018- \\
07-04\end{array}$ & $\begin{array}{l}2019- \\
07-05\end{array}$ & $\begin{array}{l}2019- \\
03-27\end{array}$ \\
\hline 14 & \begin{tabular}{|l} 
Immediat \\
e \\
maintenan \\
ce of \\
WMC by \\
filling \\
RBM in \\
void/sink \\
hole \\
\end{tabular} & $\begin{array}{l}\mathrm{SIP} / \mathrm{SQ} / 01 / \\
075-76\end{array}$ & $\begin{array}{l}\text { Prased } \\
\text { construction }\end{array}$ & $\begin{array}{l}197844 \\
8.57\end{array}$ & $\begin{array}{l}194793 \\
2.19\end{array}$ & $\begin{array}{l}1,945,0 \\
98.00\end{array}$ & $\begin{array}{l}2019- \\
06-11\end{array}$ & $\begin{array}{l}2019- \\
06-15\end{array}$ & $\begin{array}{l}2019- \\
06-13\end{array}$ \\
\hline 15 & $\begin{array}{l}\text { Immediat } \\
\text { e } \\
\text { maintenan } \\
\text { ce of } \\
\text { WMC by } \\
\text { filling } \\
\text { RBM in } \\
\text { void/sink } \\
\text { hole }\end{array}$ & $\begin{array}{l}\text { SIP/SQ/02/ } \\
075-76\end{array}$ & $\begin{array}{l}\text { Sabik } \\
\text { construction } \\
\text { pvt, Ltd. }\end{array}$ & $\begin{array}{l}196370 \\
2.83\end{array}$ & $\begin{array}{l}194740 \\
9.17\end{array}$ & $\begin{array}{l}1,912,3 \\
43.00\end{array}$ & $\begin{array}{l}2019- \\
06-11\end{array}$ & $\begin{array}{l}2019- \\
06-15\end{array}$ & $\begin{array}{l}2019- \\
06-13\end{array}$ \\
\hline 16 & $\begin{array}{l}\text { Immediat } \\
\text { e } \\
\text { maintenan } \\
\text { ce of } \\
\text { WMC by } \\
\text { filling } \\
\text { RBM in } \\
\text { void/sink } \\
\text { hole }\end{array}$ & $\begin{array}{l}\mathrm{SIP} / \mathrm{SQ} / 03 / \\
075-76\end{array}$ & $\begin{array}{l}\text { M/s. S.S. } \\
\text { Construction }\end{array}$ & $\begin{array}{l}198283 \\
9.47\end{array}$ & $\begin{array}{l}198283 \\
9.47\end{array}$ & & $\begin{array}{l}2019- \\
06-11\end{array}$ & $\begin{array}{l}2019- \\
06-15\end{array}$ & $\begin{array}{l}2019- \\
06-13\end{array}$ \\
\hline 17 & $\begin{array}{l}\text { Immediat } \\
\text { e } \\
\text { maintenan } \\
\text { ce of } \\
\text { WMC by } \\
\text { filling } \\
\text { RBM in } \\
\text { void/sink } \\
\text { hole }\end{array}$ & $\begin{array}{l}\text { SIP/SQ/04/ } \\
075-76\end{array}$ & $\begin{array}{l}\text { Bhawani } \\
\text { Nirman sewa }\end{array}$ & $\begin{array}{l}192258 \\
8.55\end{array}$ & $\begin{array}{l}190634 \\
6.49\end{array}$ & $\begin{array}{l}1,901,5 \\
54.00\end{array}$ & $\begin{array}{l}2019- \\
06-11\end{array}$ & $\begin{array}{l}2019- \\
06-15\end{array}$ & $\begin{array}{l}2019- \\
06-13\end{array}$ \\
\hline 18 & $\begin{array}{l}\text { Consultin } \\
\text { g service } \\
\text { for Detail } \\
\text { Measurem } \\
\text { ent and } \\
\text { Quality } \\
\text { Assessme } \\
\text { nt WMC } \\
17+700 \text { to } \\
34+700\end{array}$ & & $\begin{array}{l}\text { M/s Innovative } \\
\text { support Hube } \\
\text { Pvt. Ltd }\end{array}$ & $\begin{array}{l}169500 \\
0.00\end{array}$ & $\begin{array}{l}169500 \\
0.00\end{array}$ & $\begin{array}{l}169500 \\
0.00\end{array}$ & $\begin{array}{l}2019- \\
04-07\end{array}$ & $\begin{array}{l}2019- \\
07-09\end{array}$ & $\begin{array}{l}2019- \\
07-09\end{array}$ \\
\hline
\end{tabular}


Table 4: Delay Completed Contract detail

\begin{tabular}{|c|c|c|c|c|c|c|c|c|c|c|}
\hline $\begin{array}{l}\text { S. } \\
\text { N. }\end{array}$ & $\begin{array}{l}\text { Stretch } \\
\text { of Canal }\end{array}$ & $\begin{array}{l}\text { Contract } \\
\text { No }\end{array}$ & $\begin{array}{l}\text { Name of } \\
\text { the } \\
\text { contractor }\end{array}$ & $\begin{array}{l}\text { Estimate } \\
\text { d } \\
\text { Amount }\end{array}$ & $\begin{array}{l}\text { Agreeme } \\
\text { nt } \\
\text { Amount }\end{array}$ & $\begin{array}{l}\text { Total } \\
\text { Expen } \\
\text { diture }\end{array}$ & $\begin{array}{l}\text { Date of } \\
\text { signing } \\
\text { of } \\
\text { contract }\end{array}$ & $\begin{array}{l}\text { Schedu } \\
\text { le Date } \\
\text { of } \\
\text { comple } \\
\text { tion }\end{array}$ & $\begin{array}{l}\text { Compl } \\
\text { eted } \\
\text { date }\end{array}$ & Delay \\
\hline 1 & $\begin{array}{l}\text { Main dam } \\
\text { steel gate } \\
\text { fitting } \\
\text { and } \\
\text { Hydrome } \\
\text { chinacal } \\
\text { works }\end{array}$ & $\begin{array}{l}\text { SIP/HW/I } \\
\text { CB-02 }\end{array}$ & $\begin{array}{l}\text { OM Metals } \\
\text { Infra Strs } \\
\text { Pvt Ltd, } \\
\text { New Delhi, } \\
\text { India }\end{array}$ & $\begin{array}{l}2003711 \\
000\end{array}$ & $\begin{array}{l}1109277 \\
301.4\end{array}$ & $\begin{array}{l}110664 \\
0640\end{array}$ & $\begin{array}{l}2013-03- \\
19\end{array}$ & $\begin{array}{l}2016- \\
10-30\end{array}$ & $\begin{array}{l}2017- \\
09-30\end{array}$ & $\begin{array}{l}11 \\
\text { month } \\
(25.58 \\
\%)\end{array}$ \\
\hline 2 & $\begin{array}{l}\text { Ch } 0+614 \\
\mathrm{~km} \text { to ch } \\
12+037 \\
\mathrm{~km}\end{array}$ & $\begin{array}{l}\mathrm{SIP} / \\
\mathrm{MC} / \mathrm{ICB} / 0 \\
1\end{array}$ & $\begin{array}{l}\text { CTCE- } \\
\text { KALIKA } \\
\text { JV, } \\
\text { Internationa } \\
1 \text { Contractor }\end{array}$ & $\begin{array}{l}2117723 \\
000\end{array}$ & $\begin{array}{l}1670497 \\
255\end{array}$ & $\begin{array}{l}193602 \\
7123\end{array}$ & $\begin{array}{l}2009-01- \\
12\end{array}$ & $\begin{array}{l}2012- \\
01-11\end{array}$ & $\begin{array}{l}2013- \\
09-13\end{array}$ & $\begin{array}{l}8 \\
\text { month } \\
2 \text { days } \\
(22.22 \\
\%) \\
\end{array}$ \\
\hline 3 & $\begin{array}{l}\text { Ch } \\
12+037 \\
\mathrm{~km} \mathrm{ch} \\
13+000 \\
\end{array}$ & $\begin{array}{l}\text { SIP/MC/N } \\
\mathrm{CB} / 01\end{array}$ & $\begin{array}{l}\text { Tamang- } \\
\text { Rasuwa JV, } \\
\text { National } \\
\text { contractor }\end{array}$ & $\begin{array}{l}1011280 \\
00\end{array}$ & $\begin{array}{l}6500116 \\
5.15\end{array}$ & $\begin{array}{l}563035 \\
43.33\end{array}$ & $\begin{array}{l}2008-06- \\
18\end{array}$ & $\begin{array}{l}2010- \\
06-17\end{array}$ & $\begin{array}{l}2011- \\
06-26\end{array}$ & $\begin{array}{l}12 \\
\text { month } \\
9 \text { days } \\
(50 \%) \\
\end{array}$ \\
\hline 4 & $\begin{array}{l}\text { Ch } \\
13+000 \\
\text { km to ch } \\
14+000 \mathrm{k} \\
\mathrm{m}\end{array}$ & $\begin{array}{l}\text { SIP/MC/N } \\
\mathrm{CB} / 02\end{array}$ & $\begin{array}{l}\text { Waiba- } \\
\text { Bhairab- } \\
\text { Himdung \& } \\
\text { Thokar } \\
\text { JV,National } \\
\text { Contractor }\end{array}$ & $\begin{array}{l}8518000 \\
0\end{array}$ & $\begin{array}{l}5207886 \\
6.25\end{array}$ & $\begin{array}{l}427482 \\
44.63\end{array}$ & $\begin{array}{l}2008-06- \\
18\end{array}$ & $\begin{array}{l}2010- \\
06-17\end{array}$ & $\begin{array}{l}2011- \\
06-26\end{array}$ & $\begin{array}{l}12 \\
\text { month } \\
9 \text { days } \\
(50 \%)\end{array}$ \\
\hline 5 & $\begin{array}{l}\mathrm{ch} \\
14+000 \\
\mathrm{~km} \text { to ch } \\
15+000 \\
\mathrm{~km}\end{array}$ & $\begin{array}{l}\text { SIP/MC/N } \\
\mathrm{CB} / 03\end{array}$ & $\begin{array}{l}\text { Waiba- } \\
\text { Bhairab- } \\
\text { Himdung\& } \\
\text { Thokar JV, } \\
\text { National } \\
\text { Contractor. }\end{array}$ & $\begin{array}{l}8472600 \\
0\end{array}$ & $\begin{array}{l}5640709 \\
7.55\end{array}$ & $\begin{array}{l}468078 \\
24.66\end{array}$ & $\begin{array}{l}2008-06- \\
18\end{array}$ & $\begin{array}{l}2010- \\
06-17\end{array}$ & $\begin{array}{l}2011- \\
06-26\end{array}$ & $\begin{array}{l}12 \\
\text { month } \\
9 \text { days } \\
(50 \%)\end{array}$ \\
\hline 6 & $\begin{array}{l}\text { Construct } \\
\text { ion of } \\
\text { Main } \\
\text { Canal Ch. } \\
17+700 \\
\text { to } \\
35+000 \mathrm{k} \\
\mathrm{m}\end{array}$ & $\begin{array}{l}\text { SIP/MC/IC } \\
\text { B-02 }\end{array}$ & $\begin{array}{l}\text { CTCE and } \\
\text { Kalika } \\
\text { Constructio } \\
\text { n } \\
\text { P.Ltd. JV }\end{array}$ & $\begin{array}{l}3330525 \\
000\end{array}$ & $\begin{array}{l}2117566 \\
386\end{array}$ & $\begin{array}{l}302480 \\
4000\end{array}$ & $\begin{array}{l}2011-12- \\
16\end{array}$ & $\begin{array}{l}2015- \\
02-01\end{array}$ & $\begin{array}{l}2015- \\
04-15\end{array}$ & $\begin{array}{l}3 \\
\text { month } \\
(7.89 \% \\
) \text { but } \\
\text { not } \\
\text { final }\end{array}$ \\
\hline 7 & $\begin{array}{l}\text { Construct } \\
\text { ion of } \\
\text { Main } \\
\text { Canal Ch. } \\
35+000 \\
\text { to } \\
45+250 \\
\mathrm{~km}\end{array}$ & $\begin{array}{l}\text { SIP/MC/IC } \\
\text { B-03 }\end{array}$ & $\begin{array}{l}\text { Kalika/CTC } \\
\text { E }\end{array}$ & $\begin{array}{l}2032010 \\
000\end{array}$ & $\begin{array}{l}1635826 \\
332.18\end{array}$ & $\begin{array}{l}164685 \\
2000\end{array}$ & $\begin{array}{l}2015-06- \\
15\end{array}$ & $\begin{array}{l}2017- \\
12-01\end{array}$ & $\begin{array}{l}2017- \\
11-30\end{array}$ & $\begin{array}{l}\text { Constru } \\
\text { ction } \\
\text { comple } \\
\text { tes but } \\
\text { not } \\
\text { final }\end{array}$ \\
\hline 8 & $\begin{array}{l}\text { Main } \\
\text { canal } \\
\text { Protectio } \\
\text { n works }\end{array}$ & $\begin{array}{l}\text { SIP/FDR/ } \\
\text { NCB-01 }\end{array}$ & $\begin{array}{l}\text { Kalika } \\
\text { Constructio } \\
\text { n P Ltd }\end{array}$ & $\begin{array}{l}1282840 \\
00\end{array}$ & $\begin{array}{l}8518479 \\
1.23\end{array}$ & $\begin{array}{l}896300 \\
00\end{array}$ & $\begin{array}{l}2015-03- \\
01\end{array}$ & $\begin{array}{l}2015- \\
07-10\end{array}$ & $\begin{array}{l}2016- \\
04-07\end{array}$ & $\begin{array}{l}9 \\
\text { month } \\
(225 \%)\end{array}$ \\
\hline 9 & $\begin{array}{l}\text { Main } \\
\text { canal } \\
\text { maintana } \\
\text { nce work }\end{array}$ & $\begin{array}{l}\text { SIP/MC- } \\
\text { RP/NCB- } \\
01\end{array}$ & $\begin{array}{l}\text { Apex } \\
\text { Constructio } \\
\mathrm{n} \\
\text { Co.Pvt.Ltd. }\end{array}$ & $\begin{array}{l}2998400 \\
0\end{array}$ & $\begin{array}{l}1780616 \\
2.58\end{array}$ & $\begin{array}{l}167455 \\
38\end{array}$ & $\begin{array}{l}2016-06- \\
20\end{array}$ & $\begin{array}{l}2018- \\
05-13\end{array}$ & $\begin{array}{l}2018- \\
10-16\end{array}$ & $\begin{array}{l}\text { 4month } \\
3 \text { days } \\
(16.66 \\
\%) \\
\end{array}$ \\
\hline
\end{tabular}




\begin{tabular}{|c|c|c|c|c|c|c|c|c|c|c|}
\hline & $\begin{array}{l}\mathrm{ch} 0+000 \\
\text { to } \mathrm{ch} \\
25+000\end{array}$ & & & & & & & & & \\
\hline 10 & $\begin{array}{l}\text { Main } \\
\text { canala } \\
\text { gate } \\
\text { fitting } \\
\text { works }\end{array}$ & $\begin{array}{l}\mathrm{SIP} / \mathrm{MC} / \mathrm{G} \\
\text { ate/NCB- } \\
01\end{array}$ & $\begin{array}{l}\text { OM Metals } \\
\text { Infra Strs } \\
\text { Pvt Ltd }\end{array}$ & $\begin{array}{l}2330478 \\
0.7\end{array}$ & $\begin{array}{l}2330478 \\
0.67\end{array}$ & $\begin{array}{l}221279 \\
84\end{array}$ & $\begin{array}{l}2016-07- \\
12\end{array}$ & $\begin{array}{l}2018- \\
03-31\end{array}$ & $\begin{array}{l}2018- \\
07-31\end{array}$ & $\begin{array}{l}4 \\
\text { month } \\
(20 \%)\end{array}$ \\
\hline 11 & $\begin{array}{l}\text { Sighiniya } \\
\text { branch ch } \\
0+000 \text { to } \\
\text { ch } 6+000\end{array}$ & $\begin{array}{l}\text { SIP/SBC/N } \\
\text { CB-01 }\end{array}$ & $\begin{array}{l}\text { Tamang/Pac } \\
\text { ific JV, } \\
\text { Kathmandu }\end{array}$ & $\begin{array}{l}1961623 \\
85\end{array}$ & $\begin{array}{l}9320485 \\
8.08\end{array}$ & $\begin{array}{l}902235 \\
85\end{array}$ & $\begin{array}{l}2014-06- \\
19\end{array}$ & $\begin{array}{l}2016- \\
06-07\end{array}$ & $\begin{array}{l}2019- \\
02-06\end{array}$ & $\begin{array}{l}24 \text { mont } \\
\mathrm{h} \\
(100 \%)\end{array}$ \\
\hline 12 & $\begin{array}{l}\text { Sighiniya } \\
\text { sub } \\
\text { branch, } \\
\text { field } \\
\text { canal }\end{array}$ & $\begin{array}{l}\text { SIP/SBC/N } \\
\text { CB-03 }\end{array}$ & $\begin{array}{l}\text { Kankai/Tho } \\
\text { dung/Mahal } \\
\text { axmi } \\
\text { JVKtm }\end{array}$ & $\begin{array}{l}1782907 \\
03\end{array}$ & $\begin{array}{l}1242328 \\
72.77\end{array}$ & $\begin{array}{l}121365 \\
061\end{array}$ & $\begin{array}{l}2014-06- \\
17\end{array}$ & $\begin{array}{l}2016- \\
06-12\end{array}$ & $\begin{array}{l}2019- \\
07-16\end{array}$ & $\begin{array}{l}36 \\
\text { month } \\
4 \text { days } \\
(150 \%)\end{array}$ \\
\hline 13 & $\begin{array}{l}\text { Jagaiya } \\
\text { command } \\
\text { area } \\
\text { protection } \\
\text { works }\end{array}$ & $\begin{array}{l}\text { SIP/CAP/ } \\
\text { NCB-06 }\end{array}$ & M.S.-Oli JV & $\begin{array}{l}2872650 \\
0\end{array}$ & $\begin{array}{l}1977090 \\
2.62\end{array}$ & & $\begin{array}{l}2017-07- \\
16\end{array}$ & $\begin{array}{l}2018- \\
03-30\end{array}$ & $\begin{array}{l}2018- \\
07-09\end{array}$ & $\begin{array}{l}3 \\
\text { month } \\
9 \text { days } \\
(37.5 \% \\
)\end{array}$ \\
\hline 14 & $\begin{array}{l}\text { sighniyag } \\
\text { hat } \\
\text { command } \\
\text { area } \\
\text { protection } \\
\text { works }\end{array}$ & $\begin{array}{l}\text { SIP/CAP/ } \\
\text { NCB-07 }\end{array}$ & $\begin{array}{l}\text { Amar-Malla } \\
\text { JV }\end{array}$ & $\begin{array}{l}1149729 \\
36\end{array}$ & $\begin{array}{l}1059888 \\
03.6\end{array}$ & $\begin{array}{l}102388 \\
541.8\end{array}$ & $\begin{array}{l}2017-06- \\
28\end{array}$ & $\begin{array}{l}2018- \\
05-25\end{array}$ & $\begin{array}{l}2020- \\
04-07\end{array}$ & $\begin{array}{l}22 \\
\text { month } \\
12 \text { days } \\
(200 \%)\end{array}$ \\
\hline 15 & $\begin{array}{l}\text { Binonam } \\
\text { a } \\
\text { command } \\
\text { Area } \\
\text { protection } \\
\text { works }\end{array}$ & $\begin{array}{l}\text { SIP/CAP/ } \\
\text { NCB-08 }\end{array}$ & $\begin{array}{l}\text { Mahadev } \\
\text { Khimti- Oli } \\
\text { JV }\end{array}$ & $\begin{array}{l}6325971 \\
1\end{array}$ & $\begin{array}{l}4725698 \\
9.85\end{array}$ & $\begin{array}{l}468845 \\
23.75\end{array}$ & $\begin{array}{l}2017-07- \\
17\end{array}$ & $\begin{array}{l}2018- \\
04-04\end{array}$ & $\begin{array}{l}2020- \\
01-10\end{array}$ & $\begin{array}{l}21 \\
\text { month } \\
6 \text { days } \\
(233 \%)\end{array}$ \\
\hline 16 & $\begin{array}{l}\text { Construct } \\
\text { ion \& } \\
\text { Protectio } \\
\text { n work at } \\
\text { Boksini } \\
\text { khola } \\
\text { near Sikta } \\
\text { Barrage } \\
\text { Sukhar } \\
\text { khola }\end{array}$ & $\begin{array}{l}\text { SIP/CAP/ } \\
\text { NCB-13 }\end{array}$ & $\begin{array}{l}\text { M/S RAJ } \\
\text { BUILDERS } \\
\text { PVT. LTD. }\end{array}$ & $\begin{array}{l}1494100 \\
0\end{array}$ & $\begin{array}{l}8991508 . \\
00\end{array}$ & $\begin{array}{l}8,116,2 \\
44\end{array}$ & $\begin{array}{l}2018-06- \\
13\end{array}$ & $\begin{array}{l}2018- \\
04-19\end{array}$ & $\begin{array}{l}2020- \\
04-12\end{array}$ & $\begin{array}{l}23 \\
\text { month } \\
23 \text { days } \\
(120 \%)\end{array}$ \\
\hline
\end{tabular}

From table 3 it can be seen that 18 contracts were completed based on schedule because of well-planned and the performance of the contractor are good. However, the variation of cost out of 17 only 1 contract i.e., contract SIP/CAP/NCB-10(Re) has estimated cost NRs 28227187.00, agreement amount NRs 24009762.70, and expenditure NRs 24346652.00. The variation percentage of this contract has $1.4 \%$.

\subsection{Completed Contract with Delay:}

The status of delay completed 16 contract's which are shown in table 4 it clearly says the estimated cost, contract agreement amount, expenditure, agreement date, completed date, and delay percentage. From table 5 it can be seen that 16 contracts were completed based on behind the schedule because of delay due to unavailability of material, premonsoon, band, hadtal and hence performance of contractor became slow. The maximum time delay contracts are SIP/CAP/NCB-03 has 233\%, SIP/FDR/NCB-01 has $225 \%$, SIP/CAP/NCB-07 has $200 \%$, SIP/SBC/NCB-03 has $150 \%$ and so on. The average percentage of delay of the project has $82.50 \%$. The contract SIP/MC/ICB-03 has completed the construction work and final bill forwarded by the contractor but not final yet and extended the DLP 
period. The contract SIP/MC/ICB-02 has completed the construction work and final bill forwarded by contractor but not final yet and anti-graft body (CIAA) on 7th December 2018 lodged a corruption case against a contractor and 28 officials at Special Court citing misappropriation of funds and quality of construction works in Sikta Irrigation Project. Out of 16 contracts, only 4 contracts have variation. The variations of contracts are SIP/MC/ICB-01 has 15.89\%, SIP/MC/ICB-02 has 42.84\%, SIP/MC/ICB-03 has $0.67 \%$ and SIP/FRD/NCB-01 has $5.22 \%$.

\subsection{Ongoing Contracts Status:}

Out of a total of 52 contracts, only 18 contracts were completed based on schedule time whereas 16 contracts behind the schedule. However, 16 contracts are still ongoing far behind the schedule. The status of ongoing far behind schedule 16 contract's which estimated cost, agreement amount, expenditure, date of signing of contract, scheduled date, and performance of work progress. Which are shown in table 5 .

Table 5: Ongoing contract detail

\begin{tabular}{|c|c|c|c|c|c|c|c|c|c|c|}
\hline $\begin{array}{l}\text { S. } \\
\text { N. }\end{array}$ & $\begin{array}{l}\text { Stretch } \\
\text { of Canal }\end{array}$ & $\begin{array}{l}\text { Contract } \\
\text { No }\end{array}$ & $\begin{array}{l}\text { Name of } \\
\text { the } \\
\text { contracto } \\
\mathbf{r}\end{array}$ & $\begin{array}{l}\text { Estim } \\
\text { ated } \\
\text { Amou } \\
\text { nt }\end{array}$ & $\begin{array}{l}\text { Agree } \\
\text { ment } \\
\text { Amou } \\
\text { nt }\end{array}$ & $\begin{array}{l}\text { Total } \\
\text { Expen } \\
\text { diture }\end{array}$ & $\begin{array}{l}\text { Date } \\
\text { of } \\
\text { signi } \\
\text { ng of } \\
\text { contr } \\
\text { act }\end{array}$ & $\begin{array}{l}\text { Sched } \\
\text { ule } \\
\text { Date } \\
\text { of } \\
\text { comp } \\
\text { letion }\end{array}$ & $\begin{array}{l}\text { Comp } \\
\text { leted } \\
\text { date }\end{array}$ & $\begin{array}{l}\text { Perfor } \\
\text { mance }\end{array}$ \\
\hline 1 & $\begin{array}{l}\text { Purbi } \\
\text { Mul } \\
\text { nahar } \\
\text { sakha, } \\
\text { upsakha } \\
\text { atha } \\
\text { Sanrachn } \\
\text { a Nirman } \\
\text { karya. }\end{array}$ & $\begin{array}{l}\text { SIP/EMC/I } \\
\text { CB-01 }\end{array}$ & $\begin{array}{l}\text { Kalika \& } \\
\text { Kumar JV }\end{array}$ & $\begin{array}{l}38577 \\
70000\end{array}$ & $\begin{array}{l}306985 \\
9259\end{array}$ & $\begin{array}{l}16582 \\
29197\end{array}$ & $\begin{array}{l}2017- \\
07-24\end{array}$ & $\begin{array}{l}2019- \\
11-11\end{array}$ & $\begin{array}{l}\text { ON } \\
\text { GOIN } \\
\text { G }\end{array}$ & $30 \%$ \\
\hline 2 & $\begin{array}{l}\text { Sighiniy } \\
\text { a branch } \\
\text { ch } 6+000 \\
\text { to ch } \\
19+000\end{array}$ & $\begin{array}{l}\text { SIP/SBC/N } \\
\text { CB-02 }\end{array}$ & $\begin{array}{l}\text { Raman/K } \\
\text { S JV, } \\
\text { Dhangadh } \\
\text { i, Kailali }\end{array}$ & $\begin{array}{l}17120 \\
5647.9\end{array}$ & $\begin{array}{l}105469 \\
170.32\end{array}$ & $\begin{array}{l}70166 \\
502.13\end{array}$ & $\begin{array}{l}2014- \\
06-12\end{array}$ & $\begin{array}{l}2016- \\
06-04\end{array}$ & $\begin{array}{l}\text { ON } \\
\text { GOIN } \\
\text { G }\end{array}$ & $65 \%$ \\
\hline 3 & $\begin{array}{l}\text { Chater } \\
\text { tatha } \\
\text { sighiniya } \\
\text { comman } \\
\text { d area } \\
\text { p.works }\end{array}$ & $\begin{array}{l}\text { SIP/CAP/N } \\
\text { CB-02 }\end{array}$ & $\begin{array}{l}\text { Pappu } \\
\text { Constructi } \\
\text { ons, } \\
\text { Tinkune, } \\
\text { Kathmand } \\
\text { u }\end{array}$ & $\begin{array}{l}94596 \\
000\end{array}$ & $\begin{array}{l}763771 \\
66.52\end{array}$ & $\begin{array}{l}39368 \\
649\end{array}$ & $\begin{array}{l}2015- \\
03-01\end{array}$ & $\begin{array}{l}2017- \\
06-29\end{array}$ & $\begin{array}{l}\text { ON } \\
\text { GOIN } \\
\text { G }\end{array}$ & $52 \%$ \\
\hline 4 & $\begin{array}{l}\text { Chater } \\
\text { tatha } \\
\text { sighiniya } \\
\text { comman } \\
\text { d area p. } \\
\text { works }\end{array}$ & $\begin{array}{l}\text { SIP/CAP/N } \\
\text { CB-04 }\end{array}$ & $\begin{array}{l}\text { Dev and } \\
\text { Sayar- } \\
\text { Malla JV, } \\
\text { Narayan, } \\
\text { Dailekh }\end{array}$ & $\begin{array}{l}52500 \\
000\end{array}$ & $\begin{array}{l}416584 \\
12.94\end{array}$ & $\begin{array}{l}40192 \\
159\end{array}$ & $\begin{array}{l}2017- \\
06-21\end{array}$ & $\begin{array}{l}2018- \\
07-18\end{array}$ & $\begin{array}{l}\text { ON } \\
\text { GOIN } \\
\text { G }\end{array}$ & $96 \%$ \\
\hline 5 & $\begin{array}{l}\text { Construc } \\
\text { tion of } \\
\text { Protectio } \\
\mathrm{n} \text { work at } \\
\text { Dunduw } \\
\text { a Khola } \\
\text { in } \\
\text { Sidhaniy } \\
\text { a }\end{array}$ & $\begin{array}{l}\text { SIP/CAP/N } \\
\text { CB-12 }\end{array}$ & $\begin{array}{l}\text { M/S } \\
\text { NISHAN } \\
\text { BUILDE } \\
\text { RS PVT. } \\
\text { LTD. }\end{array}$ & $\begin{array}{l}18609 \\
000\end{array}$ & $\begin{array}{l}130720 \\
00.00\end{array}$ & $\begin{array}{l}5,739, \\
092\end{array}$ & $\begin{array}{l}2018- \\
06-04\end{array}$ & $\begin{array}{l}2019- \\
04-10\end{array}$ & $\begin{array}{l}\mathrm{ON} \\
\text { GOIN } \\
\mathrm{G}\end{array}$ & $44 \%$ \\
\hline 6 & $\begin{array}{l}\text { Comman } \\
\text { d Area } \\
\text { Protectio }\end{array}$ & $\begin{array}{l}\text { SIP/CAP/N } \\
\text { CB-15(Re) }\end{array}$ & $\begin{array}{l}\text { M/S } \\
\text { AMAR / }\end{array}$ & $\begin{array}{l}23694 \\
890\end{array}$ & $\begin{array}{l}189342 \\
58.70\end{array}$ & $\begin{array}{l}7,746, \\
272\end{array}$ & $\begin{array}{l}2018- \\
07-06\end{array}$ & $\begin{array}{l}\text { 5-Jul- } \\
19\end{array}$ & $\begin{array}{l}\text { ON } \\
\text { GOIN } \\
\text { G }\end{array}$ & $50 \%$ \\
\hline
\end{tabular}




\begin{tabular}{|c|c|c|c|c|c|c|c|c|c|c|}
\hline & $\begin{array}{l}\mathrm{n} \text { Works } \\
\text { at } \\
\text { Jhijhari } \\
\text { Khola }\end{array}$ & & $\begin{array}{l}\text { MAJDOO } \\
\text { R JV }\end{array}$ & & & & & & & \\
\hline 7 & $\begin{array}{l}\text { Comman } \\
\text { d Area } \\
\text { Protectio } \\
\text { n Work } \\
\text { at Rapti } \\
\text { River } \\
2700 \mathrm{~m} \\
\text { Downstr } \\
\text { eam } \\
\text { from } \\
\text { Sidhaniy } \\
\text { a Ghat } \\
\text { Bridge }\end{array}$ & $\begin{array}{l}\text { SIP/CAP/N } \\
\text { CB-16 }\end{array}$ & $\begin{array}{l}\text { M/S DEV } \\
\& \\
\text { SAYAR / } \\
\text { MALLA } \\
\text { JV }\end{array}$ & $\begin{array}{l}94803 \\
382\end{array}$ & $\begin{array}{l}845614 \\
37.45\end{array}$ & $\begin{array}{l}24,753 \\
, 045\end{array}$ & $\begin{array}{l}2018- \\
07-05\end{array}$ & $\begin{array}{l}2019- \\
07-05\end{array}$ & $\begin{array}{l}\text { ON } \\
\text { GOIN } \\
\text { G }\end{array}$ & $29 \%$ \\
\hline 8 & $\begin{array}{l}\text { Drainage } \\
\text { Develop } \\
\text { ment } \\
\text { work at } \\
\text { Pidari } \\
\text { Nala }\end{array}$ & $\begin{array}{l}\mathrm{SIP} / / \mathrm{DR} / \mathrm{NC} \\
\mathrm{B}-01\end{array}$ & $\begin{array}{l}\text { M/S } \\
\text { AMAR } \\
\text { CONSTR } \\
\text { UCTION }\end{array}$ & $\begin{array}{l}84231 \\
000\end{array}$ & $\begin{array}{l}711730 \\
00.00\end{array}$ & $\begin{array}{l}53,007 \\
, 569\end{array}$ & $\begin{array}{l}2018- \\
07-02\end{array}$ & $\begin{array}{l}2019- \\
07-03\end{array}$ & $\begin{array}{l}\text { ON } \\
\text { GOIN } \\
\text { G }\end{array}$ & $74 \%$ \\
\hline 9 & $\begin{array}{l}\text { Drainage } \\
\text { Develop } \\
\text { ment } \\
\text { work at } \\
\text { Kiran } \\
\text { Nala \& } \\
\text { Junction }\end{array}$ & $\begin{array}{l}\text { SIP//DR/NC } \\
\text { B-02 }\end{array}$ & $\begin{array}{l}\text { M/S } \\
\text { BAJRGU } \\
\text { RU } \\
\text { CONSTR } \\
\text { UCTION }\end{array}$ & $\begin{array}{l}94914 \\
000\end{array}$ & $\begin{array}{l}770960 \\
00.00\end{array}$ & $\begin{array}{l}34,083 \\
, 896\end{array}$ & $\begin{array}{l}2018- \\
07-02\end{array}$ & $\begin{array}{l}2019- \\
07-03\end{array}$ & $\begin{array}{l}\text { ON } \\
\text { GOIN } \\
\text { G }\end{array}$ & $44 \%$ \\
\hline $\begin{array}{l}1 \\
\mathbf{0}\end{array}$ & $\begin{array}{l}\text { Construc } \\
\text { tion of } \\
\text { Canal } \\
\text { and } \\
\text { Structure } \\
\text { s of } \\
\text { Akalghar } \\
\text { wa } \\
\text { Secondar } \\
\text { y Canal } \\
\text { (Ch. } \\
0+000 \text { to } \\
4+600) \\
\end{array}$ & $\begin{array}{l}\text { SIP/RMC/A } \\
\text { KL/NCB-01 }\end{array}$ & $\begin{array}{l}\text { M/S } \\
\text { BHAIRA } \\
\text { B } \\
\text { CONSTR } \\
\text { UCTION }\end{array}$ & $\begin{array}{l}87551 \\
851\end{array}$ & $\begin{array}{l}767263 \\
56.33\end{array}$ & $\begin{array}{l}31849 \\
362.08\end{array}$ & $\begin{array}{l}2018- \\
07-03\end{array}$ & $\begin{array}{l}2020- \\
07-05\end{array}$ & $\begin{array}{l}\text { ON } \\
\text { GOIN } \\
\text { G }\end{array}$ & $42 \%$ \\
\hline $\begin{array}{l}1 \\
1\end{array}$ & $\begin{array}{l}\text { Construc } \\
\text { tion of } \\
\text { Canal } \\
\text { and } \\
\text { Structure } \\
\text { s of } \\
\text { Gohawa } \\
\text { Sub- } \\
\text { Secondar } \\
\text { y Canal } \\
\text { (Ch. } \\
0+000 \text { to } \\
2+050) \\
\end{array}$ & $\begin{array}{l}\text { SIP/RMC/G } \\
\text { HW/NCB- } \\
01\end{array}$ & $\begin{array}{l}\text { M/S } \\
\text { BHAIRA } \\
\text { B } \\
\text { CONSTR } \\
\text { UCTION }\end{array}$ & $\begin{array}{l}78628 \\
461\end{array}$ & $\begin{array}{l}689998 \\
04.34\end{array}$ & $\begin{array}{l}34083 \\
896.17\end{array}$ & $\begin{array}{l}2018- \\
07-03\end{array}$ & $\begin{array}{l}2020- \\
07-05\end{array}$ & $\begin{array}{l}\text { ON } \\
\text { GOIN } \\
\text { G }\end{array}$ & $49 \%$ \\
\hline
\end{tabular}




\begin{tabular}{|c|c|c|c|c|c|c|c|c|c|c|}
\hline 12 & $\begin{array}{l}\text { Constructio } \\
\mathrm{n} \text { of Canal } \\
\text { and } \\
\text { Structures of } \\
\text { Persenipur } \\
\text { Secondary } \\
\text { Canal (Ch. } \\
0+000 \text { to } \\
4+750)\end{array}$ & $\begin{array}{l}\text { SIP/RMC } \\
\text { /PRS/NC } \\
\text { B-01 }\end{array}$ & $\begin{array}{l}\text { M/S } \\
\text { AMAR } \\
\text { CONST } \\
\text { RUCTIO } \\
\text { N }\end{array}$ & $\begin{array}{l}8661 \\
6082\end{array}$ & $\begin{array}{l}735848 \\
04.59\end{array}$ & $\begin{array}{l}211390 \\
28.28\end{array}$ & $\begin{array}{l}2018- \\
07-03\end{array}$ & $\begin{array}{l}2020- \\
07-05\end{array}$ & $\begin{array}{l}\text { ON } \\
\text { GOI } \\
\text { NG }\end{array}$ & $29 \%$ \\
\hline 13 & $\begin{array}{l}\text { Constructio } \\
\mathrm{n} \text { of Canal } \\
\text { and } \\
\text { Structures of } \\
\text { Pidari } \\
\text { Secondary } \\
\text { Canal (Ch. } \\
0+000 \text { to } \\
4+100)\end{array}$ & $\begin{array}{l}\text { SIP/RMC } \\
\text { /PDR/NC } \\
\text { B-01 }\end{array}$ & $\begin{array}{l}\text { M/S } \\
\text { MAHAD } \\
\text { EV } \\
\text { KHIMTI } \\
\text { / OLI JV }\end{array}$ & $\begin{array}{l}7444 \\
8200\end{array}$ & $\begin{array}{l}760583 \\
40.15\end{array}$ & $\begin{array}{l}102509 \\
03.67\end{array}$ & $\begin{array}{l}2018- \\
07-03\end{array}$ & $\begin{array}{l}2020- \\
07-05\end{array}$ & $\begin{array}{l}\text { ON } \\
\text { GOI } \\
\text { NG }\end{array}$ & $13 \%$ \\
\hline 14 & $\begin{array}{l}\text { Constructio } \\
\mathrm{n} \text { of Canal } \\
\text { and } \\
\text { Structures of } \\
\text { Sidhaniya } \\
\text { Secondary } \\
\text { Canal (Ch. } \\
5+200 \text { to } \\
6+000) \\
\end{array}$ & $\begin{array}{l}\text { SIP/SBC/ } \\
\text { NCB-04 }\end{array}$ & $\begin{array}{l}\text { Amar- } \\
\text { Majdoor } \\
\text { J/V }\end{array}$ & $\begin{array}{l}5548 \\
8000\end{array}$ & $\begin{array}{l}32,254, \\
078\end{array}$ & $\begin{array}{l}586277 \\
3.02\end{array}$ & $\begin{array}{l}2019- \\
05-19\end{array}$ & $\begin{array}{l}2020- \\
05-18\end{array}$ & $\begin{array}{l}\text { ON } \\
\text { GOI } \\
\text { NG }\end{array}$ & $18 \%$ \\
\hline 15 & $\begin{array}{l}\text { Maintenanc } \\
\text { e \& } \\
\text { Protecton } \\
\text { work of } \\
\text { Headworks } \\
\text { of Dunduwa } \\
\text { Irrigation } \\
\text { System }\end{array}$ & $\begin{array}{l}\text { SIP/DIS/ } \\
\text { NCB- } \\
01 / 076- \\
077\end{array}$ & $\begin{array}{l}\mathrm{M} / \mathrm{s} \\
\text { Dynamic } \\
- \\
\text { Narayana } \\
\mathrm{m} \mathrm{JV}\end{array}$ & $\begin{array}{l}5798 \\
1000\end{array}$ & $\begin{array}{l}54,128, \\
000\end{array}$ & $\begin{array}{l}146190 \\
00\end{array}$ & $\begin{array}{l}2020- \\
02-16\end{array}$ & $\begin{array}{l}2021- \\
02-22\end{array}$ & $\begin{array}{l}\text { ON } \\
\text { GOI } \\
\text { NG }\end{array}$ & $27 \%$ \\
\hline 16 & $\begin{array}{l}\text { Review of } \\
\text { Design, } \\
\text { Constructio } \\
\mathrm{n} \\
\text { Supervision } \\
\text { \& Contract } \\
\text { management } \\
\text { of East } \\
\text { Main Canal } \\
\text { of Sikta } \\
\text { Irrigation } \\
\text { Project. }\end{array}$ & $\begin{array}{l}\text { SIP/EMC } \\
\text { /Consulta } \\
\text { ncy-01 }\end{array}$ & $\begin{array}{l}\mathrm{M} / \mathrm{s} \\
\mathrm{CMS} \\
\text { Engineeri } \\
\text { ng } \\
\text { Consulta } \\
\text { ncy-full } \\
\text { Bright } \\
\text { consultan } \\
\text { cy Pvt } \\
\text { Ltd. and } \\
\text { Expert } \\
\text { professio } \\
\text { nals Pvt. } \\
\text { Ltd }\end{array}$ & $\begin{array}{l}2400 \\
000\end{array}$ & $\begin{array}{l}2,366,8 \\
00\end{array}$ & $\begin{array}{l}159720 \\
0\end{array}$ & $\begin{array}{l}2018- \\
05-17\end{array}$ & $\begin{array}{l}2020- \\
12-20\end{array}$ & $\begin{array}{l}\text { ON } \\
\text { GOI } \\
\text { NG }\end{array}$ & $67 \%$ \\
\hline
\end{tabular}

The status of 16 running contract detail as shown in this table 4 .

\section{Terminated Contracts:}

The termination contact of Dunduwa Sinchai Parnali contract no SIP/DIS/MC/ICB-01 detail shown in table 6.

Table 6: Contract Status of Terminated of Contract

\begin{tabular}{|l|l|l|l|l|l|l|l|}
\hline S.N. & $\begin{array}{l}\text { Stretch } \\
\text { of Canal }\end{array}$ & $\begin{array}{l}\text { Contrac } \\
\text { t No }\end{array}$ & $\begin{array}{l}\text { Estimated } \\
\text { Amount }\end{array}$ & $\begin{array}{l}\text { Name of the Agreement } \\
\text { contractor Amount }\end{array}$ & $\begin{array}{l}\text { Total } \\
\text { Expenditure }\end{array}$ & $\begin{array}{l}\text { Date of } \\
\text { signing of } \\
\text { contract }\end{array}$ & $\begin{array}{l}\text { Schedule } \\
\text { Date of } \\
\text { completion }\end{array}$ \\
\hline
\end{tabular}




\begin{tabular}{|c|c|c|c|c|c|c|c|c|}
\hline 1 & $\begin{array}{l}\text { Dunduwa } \\
\text { sinchai } \\
\text { Pranali }\end{array}$ & $\begin{array}{l}\text { SIP/DIS/ } \\
\mathrm{MC} / \mathrm{ICB} \\
-01\end{array}$ & 1550667301 & $\begin{array}{l}\text { Coastal/Pappu } \\
\text { JV }\end{array}$ & $\begin{array}{l}988118268 . \\
2\end{array}$ & 14903570 & $\begin{array}{l}2016-06- \\
20\end{array}$ & 2018-10-08 \\
\hline
\end{tabular}

The status of termination of contract SIP/DIS/MC/ICB-01, Contractor Name Coastal Pappu JV has agreement amount NRs 988118268 .21, agreement date 20th July 2016 and schedule date of completion 10th October 2018. But total expenditure NRs 14903570.00, i.e,, till schedule dated of completion. So, the contractor fails to complete the construction works within schedule date. Then the employer i.e., SIP has terminated the contract.

Status in terms of Budget Expenditure Based on Master plan (2007):

Table 7: Detail of Propose budget, allocated budget and expenditure of Master Plan 2007

\begin{tabular}{|r|c|r|r|r|r|}
\hline S.N. & F.Y. & $\begin{array}{c}\text { Proposed budget in } \\
\text { master plan (in } \\
\text { thousands) }\end{array}$ & $\begin{array}{c}\text { Allocated } \\
\text { Budget (in } \\
\text { thousands) }\end{array}$ & $\begin{array}{c}\text { Expenditure (in } \\
\text { thousands) }\end{array}$ & $\begin{array}{c}\text { Percentage of } \\
\text { Expenditure (\%) }\end{array}$ \\
\hline 1 & $2061-062$ & & 257547 & 26540 & 10.30 \\
\hline 2 & $2062-063$ & 245000 & 269450 & 257564 & 95.59 \\
\hline 3 & $2063-064$ & 532000 & 600524 & 596509 & 99.33 \\
\hline 4 & $2064-065$ & 1193500 & 767350 & 760950 & 99.17 \\
\hline 5 & $2065-066$ & 2016500 & 1078987 & 1075844 & 99.71 \\
\hline 6 & $2066-067$ & 1823000 & 1366295 & 1361619 & 99.66 \\
\hline 7 & $2067-068$ & 1671000 & 1284706 & 1276289 & 99.34 \\
\hline 8 & $2068-069$ & 2083000 & 992308 & 989492 & 99.72 \\
\hline 9 & $2069-070$ & 2009800 & 1605518 & 1552974 & 96.73 \\
\hline 10 & $2070-071$ & 1228000 & 1142069 & 1138957 & 99.73 \\
\hline & Total & $\mathbf{1 2 8 0 1 8 0 0}$ & $\mathbf{9 3 6 4 7 5 4}$ & $\mathbf{9 0 3 6 7 3 8}$ & $\mathbf{9 6 . 5 0}$ \\
\hline
\end{tabular}

(Source: SIP audit Report, 2018) [12]

The project was started 2063/064 and have a 9 year for complication time i.e, 2072/073. Total project cost has total project cost was NRs12801739074.00 but not complete in timely. According to Master Plan 2007 the proposed budget has NRs 12801800000 but allocated only budget NRs 9364754000 i.e,, $73.15 \%$ and expenditure NRs 9036738000 . i.e, $96.50 \%$. up to f.y. $2072 / 073$.

From the figure 1 clearly shows that facial year 2061-062 to f.y.2063-064 of the allocated budget more than the approved proposed budget in master plan. The expenditure is 1 st year $10.20 \%$, 2nd yr $95.59 \%$ and 3rd year 99.33\%. After that F.Y. 2064-065 to F.Y. 2070-071 allocated budget less than the proposed budget in master plan and expenditure more than $99 \%$. So, it shows that the project was delay due to financial shortage. 


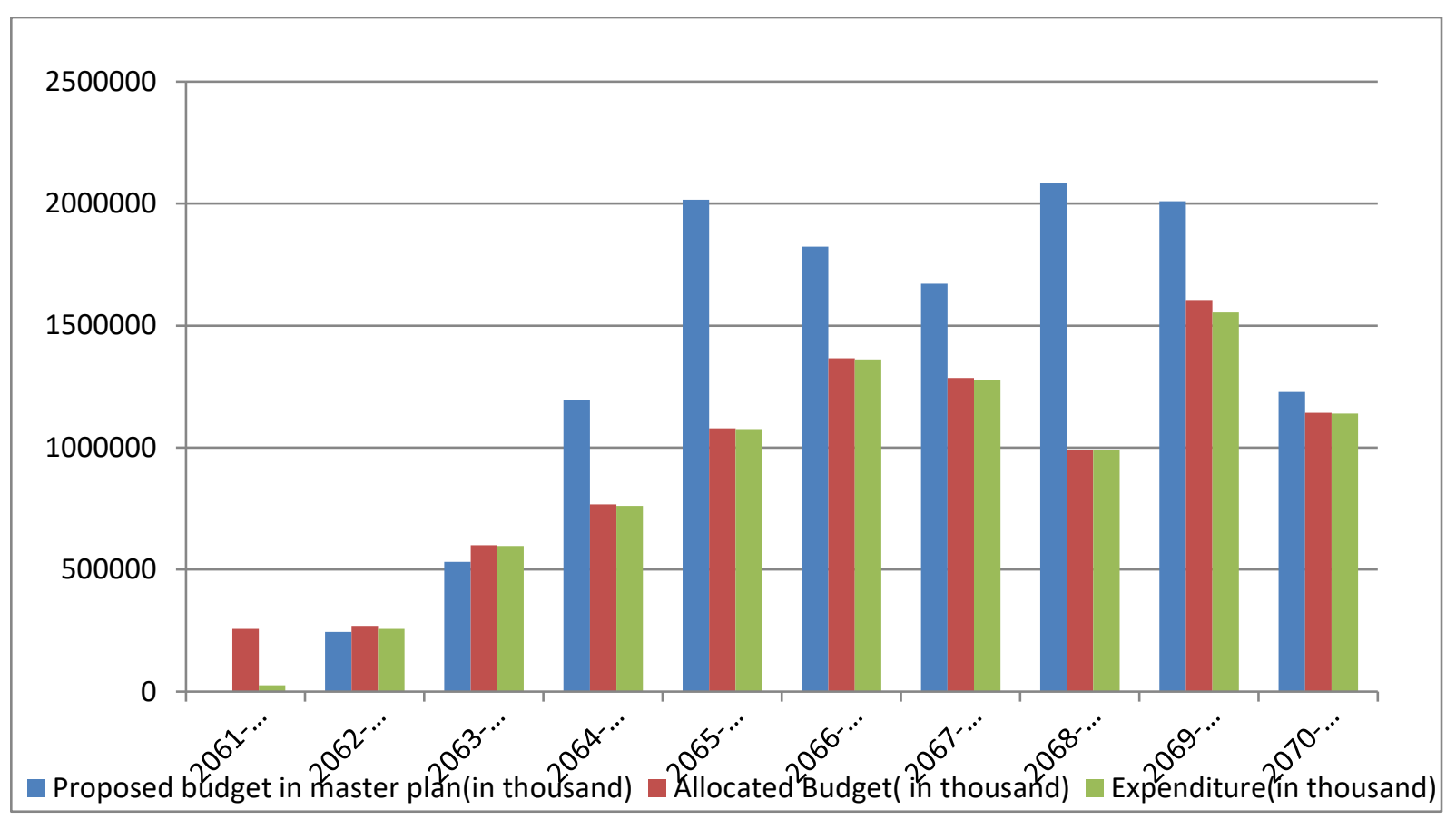

Fig. 1 : Bar chart for detail of propose budget, allocated budget and expenditure of Master Plan 2007

\section{Status in Terms of Budget Expenditure Based on Revised Master Plan 2014 :}

Table 8: Detail of Propose budget, allocated budget and expenditure of Master Plan 2007

\begin{tabular}{|r|c|r|r|r|r|}
\hline S.N. & F.Y. & $\begin{array}{c}\text { Proposed budget } \\
\text { in master plan }\end{array}$ & $\begin{array}{c}\text { Allocated } \\
\text { Budget (in } \\
\text { thousands) }\end{array}$ & $\begin{array}{c}\text { Expenditure } \\
\text { (in } \\
\text { thousands) }\end{array}$ & $\begin{array}{c}\text { Percentage of } \\
\text { Expenditure } \\
\text { (\%) }\end{array}$ \\
\hline 1 & $2061-062$ & & 257547 & 26540 & 10.30 \\
\hline 2 & $2062-063$ & 269450 & 269450 & 257564 & 95.59 \\
\hline 3 & $2063-064$ & 600500 & 600524 & 596509 & 99.33 \\
\hline 4 & $2064-065$ & 767400 & 767350 & 760950 & 99.17 \\
\hline 5 & $2065-066$ & 1078987 & 1078987 & 1075844 & 99.71 \\
\hline 6 & $2066-067$ & 1371289 & 1366295 & 1361619 & 99.66 \\
\hline 7 & $2067-068$ & 1284704 & 1284706 & 1276289 & 99.34 \\
\hline 8 & $2068-069$ & 992308 & 992308 & 989492 & 99.72 \\
\hline 9 & $2069-070$ & 1605518 & 1605518 & 1552974 & 96.73 \\
\hline 10 & $2070-071$ & 1142069 & 1142069 & 1138957 & 99.73 \\
\hline 11 & $2071-072$ & 1400632 & 1600632 & 1572785 & 98.26 \\
\hline 12 & $2072-073$ & 3007510 & 1506400 & 1407850 & 93.46 \\
\hline 13 & $2073-074$ & 3192793 & 1438764 & 800895 & 55.67 \\
\hline 14 & $2074-075$ & 2928883 & 1237159 & 918264 & 74.22 \\
\hline 15 & $2075-076$ & 3316645 & 1596000 & 1472300 & 92.25 \\
\hline 16 & $2076-077$ & 2064492 & 1521663 & 1053115 & 69.21 \\
\hline & Total & $\mathbf{2 5 0 2 3 1 8 0}$ & $\mathbf{1 8 2 6 5 3 7 2}$ & $\mathbf{1 6 2 6 1 9 4 6 . 8 9}$ & 89.03 \\
\hline
\end{tabular}

(Source: SIP audit Report, 2018) [12]

In the revised master plan total cost of the project has Rs 25023180198.54 and the project was completed in F.Y.2076-077 but not complete till now. In Annex (table 8) it shows that the proposed 
budget of the approved master plan and allocated budget of this project duration has not been equal it means the allocated budget of Rs 18265372000 is less than the proposed budget of Rs 25023180000 . The expenditure at end of 2076-077 has Rs 16261946890.00 and the average expenditure of the allocated budget has $89.03 \%$.

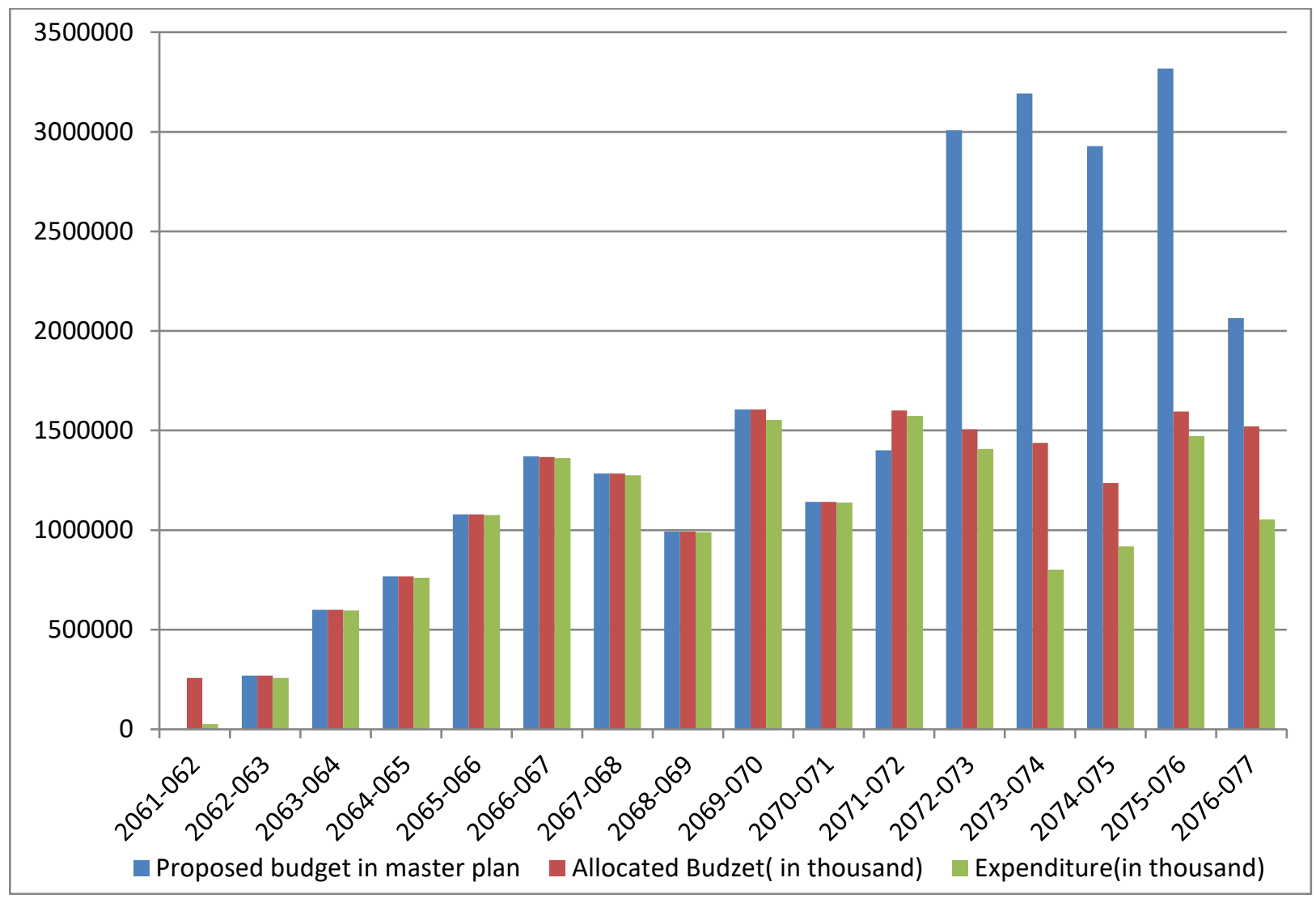

Fig. 2: Bar chart shows detail of budget allocation and expenditure (SIP, 2020)

In the above Bar chart Fig.2, it was clearly seen that in comparison of the proposed budget, the allocated budget in duration F.Y.2072/073, F.Y.2073/074, F.Y.2074/075, F.Y. 2074/075, F.Y.2075/076, F.Y.2076/077 was $0.5 \%, 0.45 \%, 0.4 \%, 0.48 \%$, and $0.73 \%$ respectively. This indicates a very less allocated budget in this period.

In comparison of the allocated budget, the expenditure in duration F.Y. 2061-062, F.Y. 2062-063 to F.Y. 2072-073, F.Y.2073-074, F.Y.2074-075, F.Y.2075-076, and F.Y. 2076-077 was 10.30\%, more than $90 \%, 55.66 \%, 74.22 \%, 92.25 \%$ and $69.21 \%$ The project starting period 2061-062 only the expenditure 10.30\%. F.Y.2073-074, F.Y.2074-075 shows that expenditure very less. In this duration quick change in the project director affects the project progress. Financially the $64.90 \%$ of the work was completed.

\section{Status in terms of Land Acquisition:}

The Sikta Irrigation Project was Land Acquisition and compensation for the land up to F.Y.2076/077 has a total $871901 \mathrm{sqm}$ and total no of plot acquisition has 1723 .

Table 9: Land Acquisition up to 2076/077

\begin{tabular}{|c|l|c|c|}
\hline S.N. & $\begin{array}{l}\text { Main Canal, Branch and Sub- } \\
\text { Branch Canal }\end{array}$ & $\begin{array}{c}\text { No of plot } \\
\text { Acquisition }\end{array}$ & $\begin{array}{c}\text { Acquisition of Land Area } \\
\text { (Sqm) }\end{array}$ \\
\hline 1 & Western Canal & 174 & 92809 \\
\hline 2 & Shighniya Secondary Canal & 649 & 224793 \\
\hline 3 & Shamsergang & 5 & 4507 \\
\hline
\end{tabular}




\begin{tabular}{|c|l|c|c|}
4 & Nepalganj office & 16 & 23230 \\
\hline 5 & Chatar & & 14392 \\
\hline 6 & Dudwa Secondary Canal & 286 & 108597 \\
\hline 7 & Eastern Main Canal & 5 & 4597 \\
\hline 8 & Akalgharuwa secondary Canal & 214 & 104030 \\
\hline 9 & Gohawa Secondary Canal & 65 & 44401 \\
\hline 10 & Persenipur Secondary Canal & 153 & 107031 \\
\hline 11 & Pidari Secondary Canal & 146 & 78082 \\
\hline 12 & Aagaiya Area & 10 & 65432 \\
\hline & Total & $\mathbf{1 7 2 3}$ & $\mathbf{8 7 1 9 0 1}$ \\
\hline
\end{tabular}

Status in terms of Discharge of Canal

The Western canal running in different dated flows of maximum discharge which are given below.

Table 10: Maximum discharge of Western Canal of SIP

\begin{tabular}{|c|c|c|c|l|}
\hline $\mathbf{S}$. & $\begin{array}{c}\text { Design Discharge } \\
\mathbf{N}\end{array}$ & $\begin{array}{c}\text { Measured Max. } \\
\text { Discharge }\left(\mathbf{m}^{\mathbf{3}} / \mathbf{s}\right)\end{array}$ & \multicolumn{1}{|c|}{ Date } & \multicolumn{1}{|c|}{ Remarks } \\
\hline 1 & 50 & 15.0 & June, 2016 & $\begin{array}{l}\text { Fails (Break) Canal during Trial } \\
\text { Testing }\end{array}$ \\
\hline 2 & 50 & 5.85 & June, 2018 & Canal running \\
\hline 3 & 50 & 13.40 & July, 2018 & Second time canal fails( Break) \\
\hline 4 & 50 & 1.46 & Jan., 2019 & Canal running \\
\hline 5 & 50 & 1.46 & May, 2020 & Canal running \\
\hline 6 & 50 & 4.14 & June, 2020 & Canal running \\
\hline 7 & 50 & 4.14 & July, 2020 & Canal running \\
\hline 8 & 50 & 2.67 & Aug., 2020 & Canal running \\
\hline
\end{tabular}

(Source: SIP Barrage control Room Record, 2020)

From table 10 it is seen that design discharge of western canal 50.00 cumecs and measured maximum discharge 15 cumecs of 27th June 2016 of canal failure at the time of trial testing is an example of a technical problem. Canal fails at Changai Nala during the Second time 20th July 2018 measured discharge 13.4 cumecs. It clearly says that the canal has not to test full design discharge. The anti-graft body (CIAA) on 7th December 2018 lodged a corruption case against the Contractor and 28 officials at Special Court citing misappropriation of funds in Sikta Irrigation Project.

\section{CONCLUSION :}

Sikta Irrigation Project has a total of 52 contracts out of which consider for study based on documentation, only 18 contracts were completed based on schedule time whereas 16 contracts behind the schedule. However, 16 contracts are still ongoing far behind the schedule and one contract terminated. According to the master plan of 2007, the total estimated cost of the project was NRs 12.80 billion and project completion date 2014/015 (B.S.2071/072), i.e., 9 years. During this period total allocated budget was 9.36 billion out of which expenditure was 9.03 billion. After that, the scope of work was added and revised the master plan with a total estimated project cost of 25.02 billion (2014) with the extension of time till 2019/020 (B.S.2076/77). As of the date 2019/020 (2076/077), only an 18.26 billion (72.99\%) budget was found to be allocated with an expenditure of 16.26 billion. It indicates the progress of the project was $64.90 \%$ and fails to complete the project in scheduled time 2019/020 (B.S.2076/077).

\section{ACKNOWLEDGEMENT :}

Thank God for giving such a great ability to Er. Prakash Yadav for staying at the site and working such hard and some part of it used for his master's thesis also. 


\section{REFERENCES}

[1] Mishra, A. K., Yadav, P. \& Aithal, P. S., (2021). Dispute of the Contracts: A Case from Sikta Irrigation Project, Banke, Nepal. International Journal of Case Studies in Business, IT, and Education (IJCSBE), 5(1), 169-185.

[2] Mishra, A. K., \& Bhandari, E. S. (2018). Performance assessment of ongoing construction projects under town development fund, Nepal. International Journal of Advanced Research in Civil \& Structural Engineering, 1(1\&2), 27-39.

[3] Mishra, A. K., (2019). Implementation Status of Value Management in Project Management Practice in Nepal. International Journal of Management Studies, 6(1), 2231-2528.

[4] Department of Water Resource and Irrigation Annual book f.y.2075/076, Department of Water Resource and Irrigation, Kathmandu Nepal. https://dwri.gov.np

[5] Sikta Irrigation Project Master Plan 2007 and 2014 and 2019, Nepalgunj, Banke. https://dwri.gov.np

[6] Mishra, A. K., \& Aithal, P. S. (2020). Financial Impact Assessment of Time Overrun: A Case of Second Small Towns Water Supply and Sanitation Sector Project. Nepal International Journal of Applied Engineering and Management Letters (IJAEML), 4(2), 159-173.

[7] Mishra, A. K., Sudarsan, J. S. \& Nithiyanantham, S. (2020). Assessment of time-cost model of public health buildings in Nepal. Asian J. Civ Eng. 1-10. https://doi.org/10.1007/s42107-02000294-4.

[8] Memon, A. H., Rahman, I. A., Akram, M., \& N. Md. (2014). Significant Factors Causing Time Overrun in Construction Projects of Peninsular Malaysia. University Tun Hussein Onn Malaysia, Malaysia.

[9] Suwal, A., Shrestha, S. K. (2016). Causes of Delays of Motorable Bridge Construction Under Postal Highway Projects, Departments of Roads. Journal of Advanced College of Engineering and Management, 2(1), 85-92.

[10] Yadav, S. K., \& Mishra, A. K. (2019). Status of Time and Cost Overrun of Health Building Construction Projects in Nepal. Sch J. Eng Tech, 7(9), 262-270.

[11] Sikta Irrigation Project DPR 2014, Nepalgunj, Banke. https://dwri.gov.np

[12] Sikta Irrigation Project Audit Report, 2018. https://dwri.gov.np

$* * * * * * * * * *$ 